RESEARCH PAPER RP1272

Part of Journal of Research of the National Bureau of Standards, Volume 24, January 1940

\title{
MATHEMATICAL THEORY OF IRROTATIONAL TRANSLATION WAVES
}

\author{
By Garbis H. Keulegan and George W. Patterson
}

\section{ABSTRACT}

This paper is the first of a series dealing with the motion of flood waves and other waves of translation in open channels. The case treated is that of waves for which the forces of fluid friction are negligible with respect to the inertia and gravitational forces. The irrotational motion of a perfect liquid in a horizontal rectangular canal when the original surface is disturbed is investigated on the assumption that the horizontal velocity in a cross section is approximately uniform. The results are also applicable to motion in a canal of uniform slope containing water originally moving with a uniform velocity. Special emphasis is laid on disturbances which are propagated without change of form, and in these cases formulas are derived for the wave profile and velocity of propagation. Formulas are also derived which give the deformation, energy, motion of the center of gravity, and moment of instability of an arbitrary intumescence. Consideration is given to the maximum height of a wave of permanent form. Formulas have been compared with the available experimental data. Of special interest is the comparison of the shape of the undulations composing the head of an initial sur6e with the characteristics of the cnoidal wave.

\section{CONTENTS}

List of symbols. 48

I. Introduction

II. Irrotational motion

1. Dynamical equations of motion $\ldots \ldots \ldots 3$

2. Displacement and deformation of a liquid element_........ 54

3. Kinematical relations__._.

4. Irrotational motion _....... 56

5. Invariance of the circulation in a moving circuit

6. Impulsive generation of motion

7. Pressure equation

8. Solution of problems in irrotational motion

III. Formulation of the wave problem - Waves in water of small depth_.. 61

IV. Long waves of negligible height and curvature

1. Velocity of propagation

2. Energy of an intumescence

3. Displacement of the particles

4. Effect of an arbitrary initial disturbance

5. Reflection and transmission of waves $\ldots \ldots \ldots \ldots 7$ 
V. Wave

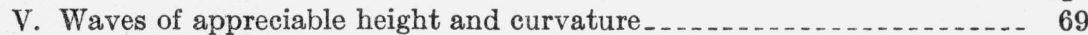

1. Two definitions of velocity of propagation ............. 69

2. Mean velocity of particles........... 70

3 . Effect of curvature and height on velocity of propagation of a volume element..................

4. Deformation of the wave profile .

5. Motion of the center of gravity of a wave

6. Variation in energy of an intumescence..................

7. The moment of instability of an intumescence

8. Solitary waves

9. Cnoidal waves

10. Moment of instability of a solitary wave

11. Moment of instability and wave formation

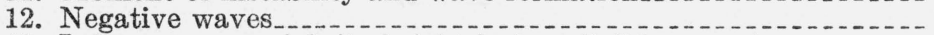

13. Intumescences of finite height but negligible curvature.......

14. Horizontal channel in communication with the ocean.........

15. Waves due to sudden increase of discharge .................

16. Effect of channel velocity distribution

17. Initial waves

18. Favre's experiments on the undulations in a positive surge

19. Theory of breakers...........

VI. References

c velocity of propagation of long wave of negligible height and curvature.

$c_{1}$ velocity of wave approaching a discontinuity in a channel cross section. Also a constant of integration.

$c_{2}$ velocity of transmitted wave after passing a discontinuity in cn $(x, k) \quad$ Jacobian elliptic function, cosine amplitude.

$\rho g E$ total energy of a wave per unit width of channel [MLT-2].

$E_{1}(k)$ complete elliptic integral of the second kind.

$f(), F(), \quad$ with or without subscripts-functional symbols.

$F(x, k)$ incomplete elliptic integral of the first kind.

$F_{1}(k)$ complete elliptic integral of the first kind.

$g$ acceleration of gravity.

$g(), G()$ functional symbol.

$h$ height of main body of discharge wave (section V-17); vertical displacement of water surface.

$h_{1}$ vertical displacement of surface as wave approaches discontinuity in channel cross section (section IV-5).

$h_{2}$ vertical displacement of surface after wave passes discontinuity in channel cross section (section IV-5).

$h_{1}$ maximum height of solitary wave, maximum depth of negative wave, maximum height of initial discharge wave.

$h_{1}, h_{2}$ maximum height and depth, respectively, of cnoidal wave.

$h_{3}=H \eta_{3}$.

$h_{a}$ maximum height of initial discharge wave.

$h^{\prime}$ average height of discharge wave.

$H$ undisturbed depth of liquid in channel.

$H($ ) functional symbol.

$k$ modulus of elliptic integral.

$l$ length of section of channel (section IV-4).

$l, m, n$ direction cosines.

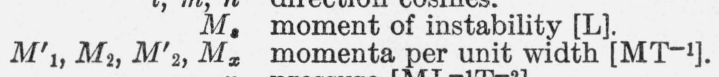

$p$ pressure $\left[\mathrm{ML}^{-1} \mathrm{~T}^{-2}\right]$.

$p_{a}$ atmospheric pressure.

$q$ speed of particle (section II-5, 7, 8).

$q$ discharge per unit width in a cross section [ $\mathrm{L}^{2} \mathrm{~T}^{-1}$ ] (section $\mathrm{V}-2$ ).

$Q$ total volume per unit width of a solitary wave [ $\left.\mathrm{L}^{2}\right]$.

$s$ length of arc. 
sn $(x, k)$ Jacobian elliptic function, sine amplitude.

$t$ time.

$u, v, w \quad x$ component, $y$ component, $z$ component, respectively, of velocity.

$u_{0} \quad x$ component of velocity at bottom of channel, also first approximation to velocity in cross section.

$u_{0}, v_{0}, w_{0}$ velocity components prior to impulse (section II-6)

$u_{1}, u_{2}$ velocities of particles in a wave before and after passing a discontinuity in a channel cross section (section IV-5).

$u_{1}, u_{2}, U_{1}, U_{2}$ local and mean velocities in two cross sections (section $\mathrm{V}-16$ ).

$u_{1}$ velocity of particle at apex of wave (section V-19).

$U$ mean velocity of particles in cross section.

$U_{0}$ mean velocity in a section where $h=0$.

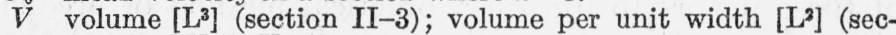
tions IV $-3, \mathrm{~V}-5)$.

$x$ Cartesian coordinate, specifically, parallel to channel in bottom plane.

y Cartesian coordinate, specifically, lateral to channel in the horizontal plane.

$z$ Cartesian coordinate, specifically, drawn upward with origin at bottom of channel.

$\alpha=3 / H^{3}$ (section $\mathrm{V}-10$ ); a ratio (section $\mathrm{V}-14$ ).

$\gamma_{1}, \gamma_{2}, \gamma_{3}$ rates of angular dilatation [ $\left.\mathrm{T}^{-1}\right]$.

$\Gamma$ circulation $\left[\mathrm{L}^{2} \mathrm{~T}^{-1}\right]$.

$\epsilon_{1}, \epsilon_{2}, \epsilon_{3}$ rates of linear dilatation [ $\left.\mathrm{T}^{-1}\right]$.

$\epsilon$ a small quantity.

$\xi, \eta, \zeta$ components of vorticity [ $\left.\mathrm{T}^{-1}\right]$ (sections II-1, 4, 5).

$\zeta$ dimensionless $x$ coordinate of free surface.

$\eta, \xi z$ and $x$ coordinates, respectively, of center of gravity of volume above undisturbed surface (sections $\mathrm{V}-5,6,12,17$ ).

$\eta$ dimensionless $z$ coordinate of free surface $=h / H$ (sections $\mathrm{V}-8$, 9); Boussinesq number (section V-16).

$\eta^{\prime}, \eta^{\prime \prime}, \eta^{\prime \prime \prime}=-\eta_{3}$ three roots of the differential equation of the permanent wave.

$\eta_{1}$ maximum relative height of a solitary wave (section $\mathrm{V}-8$ ); maximum relative height of a cnoidal wave (section $\mathrm{V}-9$, 18).

$\theta\left(\eta_{2}\right.$ maximum relative depression of a cnoidal wave.

$\lambda$ wave length of a cnoidal wave (sections $V-9,18$ ).

$\rho$ density of liquid $\left[\mathrm{ML}^{-3}\right]$.

$\sigma$ partial volume per unit width of an intumescence [ $\left.\mathrm{L}^{2}\right]$.

$\Sigma$ volume per unit width of an intumescence.

$\phi$ velocity potential [ $\left.\mathrm{L}^{2} \mathrm{~T}^{-1}\right]$

$\phi_{0}$ velocity potential prior to application of impulse (section II-6); velocity potential, first approximation.

$\psi($ ) functional symbol.

$\omega$ velocity of propagation of an element of volume of an intumescence.

velocity of propagation of an element of wave height, negligible surface curvature.

velocity of propagation of an element of wave height, appreciable surface curvature.

$\omega_{c} \quad$ velocity of propagation of a cnoidal wave.

$\omega_{Q}$ velocity of propagation of discharge.

$\omega$ impulsive pressure $\left[\mathrm{ML}^{-1} \mathrm{~T}^{-1}\right]$.

$\Omega$ gravitational potential [ $\left.\mathrm{L}^{2} \mathrm{~T}^{-2}\right]$.

\section{INTRODUCTION}

The growing importance of predicting the occurrence of floods and the rate of travel and height of flood waves as they pass down rivers has led in recent years to a marked increase in the literature on this subject. However, in no language can there be found a comprehensive presentation of the mathematical treatment of the problem of such waves. Instead, various authors at different times and in dif- 
ferent countries have treated separate aspects of the problem, so that the student of the subject must read many papers in several languages to obtain a satisfactory grasp of the problem. There is definite need for a systematic treatment of the results of these different methods of attack, presenting in coordinated form the results that have been attained up to the present time.

It is for this reason that the National Bureau of Standards, at the suggestion of the United States Weather Bureau, has commenced the preparation of a series of papers dealing with the mathematical theory of flood waves and other waves of translation. It is not the purpose of these papers to furnish a practical method of predicting the rate of flood-wave travel and the rate of attenuation of the wave in an actual river channel with its complex flow conditions. Instead, the purpose is to furnish a sound mathematical theory on which attempts to solve the practical problems of flood prediction can be based.

The present paper, the first of the series, deals with the motion of translation waves in channels of uniform, rectangular cross section when the forces of fluid friction are negligible with respect to the inertia and gravitational forces. Later papers in the series as now planned are in various stages of completion and will deal with the following topics: The effect of turbulence and channel slope and configuration on the motion of translation waves; the theory of quasipermanent regime and the methods of prediction of flood waves; and recent advances in the problem of the deformation of an intumescence.

The different regimes of the flow of water in canals and natural watercourses may be characterized as uniform, varied, or unsteady.

In the uniform regime the depth and mean velocity are invariable with respect to both time and space. This regime is attained in sufficiently long stretches of natural courses, provided the slope and discharge are constant, and the channel has a uniform cross section.

In varied flow the depth and mean velocity are unchanging with respect to time, but not with respect to space. Examples are flow in the backwater region of a dam, flow in a canal connecting reservoirs at different elevations, and flow in transition sections.

On the other hand, the unsteady regime is characterized by a depth and velocity which vary with respect to both time and space. Gravity waves and capillary waves belong to this class of phenomena, the former being the more important of the two, considered from the point of view of applied science. Gravity waves fall into two classessurface waves and translation waves. In surface waves, which occur in relatively deep waters, the motion decreases rapidly from the surface downward. The wave motion is sinusoidal, the individual particles periodically traverse closed paths, and the vertical accelerations are always appreciable. In translation waves, on the other hand, the motion of all the particles in a cross section is sensibly the same at any given instant. As a translation wave passes, the liquid particles are permanently displaced by an amount proportional to the volume of the wave. Waves of this type are frequently encountered in channels and rivers. Tidal waves are examples of the same phenomenon in seas and oceans.

Perhaps the simplest classification of translation waves is that obtained by considering the physical condition governing the motion. Two extreme cases are immediately apparent. First, we have the 
case in which the effect of friction is negligible. In these waves the volume and the length are small; and the latter fact explains why the effect of friction can be neglected, so that the weight and inertia become the factors controlling the motion. If the curvature of the wave surface is negligible, the velocity of propagation of the wave height, $\omega_{0}$, is given by the Lagrange velocity law, $\omega_{0}=\sqrt{g H}$, where $g$ is the acceleration of gravity and $H$ is the channel depth. On the other hand, if the maximum curvature and the height above the undisturbed level of the free surface are appreciable, then the velocity of propagation of the wave height $\omega_{1}$ equals $\omega_{0}$ times a factor slightly different from unity, the exact value of the factor being a function of the height and the derivatives of the wave surface. We call such disturbances potential intumescences or irrotational translation waves. They can be generated by suddenly immersing a solid body in the liquid or suddenly adding a small mass of liquid; they may also be formed by the sudden motion of a body immersed in the liquid. In these cases the disturbed position of the surface is entirely above the original surface; that is, the waves have a positive elevation. The solitary wave is a familiar example. Waves can also be produced by abruptly removing from the channel a small quantity of water or an object already in the channel. These waves have a negative elevation. Surges produced in channels by an abrupt change of flow at either end also belong in this category.

At the other extreme, we find the quasi-permanent regime of rivers, or what are commonly called flood waves. These are also translation waves, but they are waves in which friction is the all-important factor, the effects of weight and inertia being negligible. The waves are very long, which accounts for the predominant effect of friction. It is illuminating to compare flood waves and potential intumescences on the basis of their velocities of propagation. When discussing flood waves, it is convenient to utilize the idea of the velocity of propagation of discharge, $\omega_{q}$. According to the Kleitz-Seddon law $[1]^{1}$ this velocity is given by $d q / d H$, the slope of the rating curve, $q$, being the discharge per unit width occurring at the normal stage, $H$. In a rectangular channel $\omega_{q}=3 U / 2$, if the Chezy resistance law is used. A comparison of the two wave velocities gives $\omega_{0}{ }^{2} / \omega_{q}{ }^{2}=(4 / 9)\left(g H / U^{2}\right)$. Since in rivers the ratio $g H / U^{2}$ is a large number, the conclusion is that $\omega_{0}$ is many times larger than $\omega_{q}$. This difference of velocities is significant.

It should be understood that in reality there exists a continuous transition between the two extreme types of waves described above. There are other phenomena occupying an intermediate position, in which the effects of inertia, height, and friction are of the same order of magnitude. These phenomena are also of engineering interest, particularly in connection with hydroelectric plants. When the turbines of such plants are suddenly shut down, a positive intumescence is generated in the supply canal and travels upstream, increasing in length as it goes. A negative intumescence is simultaneously produced in the discharge canal, and this travels downstream, decreasing in length. These waves may be of considerable height (1 meter or so) and are attenuated by the forces of friction. Friction cannot be neglected, because of the long distances which the waves travel.

1 Figures in brackets indicate the literature references at the end of this paper. 
Similarly, when the turbines are suddenly started, the same phenomenon occurs, but the signs of the intumescences are reversed. If the pressure and velocity variations caused by these waves are large, these effects must be considered in designing channel walls, bottom, and embankments. Waves like the above, except that they are generally propagated in still water, occur in canal reaches adjacent to locks when the latter are operated. The positive and negative waves that are generated are quite long, traverse large distances, and, although they undergo a deformation of form owing to the action of friction, the wave length remains the same [2]. There are also a few special cases of flood waves occurring in mountainous regions, in which the inertia effects are important. Massé [3] has given the name of average intumescences to the phenomena described above.

The present paper deals exclusively with potential intumescences. Since friction is negligible for these waves, and since they are generated in a liquid either initially at rest or in uniform motion, then by the Lagrange-Cauchy theorem the motion of the liquid particles is irrotational. This fact justifies the mathematical approach selected for this paper.

A brief presentation of the elements of irrotational theory is given in section II, primarily to emphasize the basic dynamical and kinematical principles involved. Section III is a formulation of the basic differential equations whose solutions constitute the solution of the problem.

The general theory is applied in sections IV and V, which deal with solutions of the equations to a first and to a second approximation. In the first approximation, the height and curvature of the waves are assumed negligible, and a complete solution is found in which the waves are propagated without change of form. In the treatment of the second approximation, which constitutes the main body of the paper, more general assumptions are made, leading to involved differential equations which can be solved easily only for particular cases. The deformation of the wave profile during propagation and the motion of the center of gravity of the intumescence play an important role in this analysis. These points are discussed after the introduction of the concept of velocity of propagation of a volume element, originally due to Boussinesq. There are three integrals invariant with respect to time associated with intumescences of appreciable curvature - the volume, the energy, and the moment of instability - which are discussed in considerable detail.

The main emphasis is on the intumescence whose wave profile is propagated without change of form, and the particular solution is obtained for this case. If the liquid is undisturbed at infinity, we have the well-known solitary wave which has been investigated experimentally. If the liquid is in motion at infinity and this motion is indeterminate, we have the less familiar cnoidal waves. In the former case, there is an interesting principle discovered by Boussinesq-namely, for all intumescences of equal energy, the solitary wave is unique in possessing the minimum moment of instability. This theorem is significant in that it explains the frequent occurrence and ease of formation of the solitary wave.

In discussing the velocity of propagation of surges, an attempt is made to determine the effect of the channel velocity distribution. The characteristics of the initial wave in a surge are not without 
interest, and this wave is considered in the light of both theory and experiment.

The concluding section touches on the problem of limiting wave heights and the breaking of waves.

In the preparation of this paper, extensive use has been made of Lamb's comprehensive text [4] and of a classic paper by Boussinesq [5]. Whenever appropriate experimental data have been available, the authors have attempted to discuss the agreement between theory and experiment. The principal sources of data have been the well-known researches of Bazin [6] and Russell [7,8] and a recent paper by Favre [2].

\section{IRROTATIONAL MOTION}

\section{DYNAMICAL EQUATIONS OF MOTION}

We consider an elementary parallelepiped $\delta x \delta y \delta z$ having its corner nearest the origin of the rectangular axes of reference at the point $(x, y, z)$. Designating the component velocities at the point $(x, y, z)$ by $u, v, w$, and the density of the liquid by $\rho$, we have for the kinetic reaction in the $x$ direction of the liquid in the parallelepiped

$$
\rho \frac{d u}{d t} \delta x \delta y \delta z,
$$

which is equal to the $x$ component of all the forces acting on the liquid in the parallelepiped. If the pressure at the point $(x, y, z)$ is $p$, we have for the $x$ component of the force due to pressure,

$$
-\frac{\partial p}{\partial x} \delta x \delta y \delta z \text {. }
$$

Denoting the potential of gravity at $(x, y, z)$ by $\Omega$, we have for the $x$ component of force due to the weight of liquid,

$$
-\rho \frac{\partial \Omega}{\partial x} \delta x \delta y \delta z \text {. }
$$

Equating eq 1 to eq 2 plus eq 3 , dividing by $\rho \delta x \delta y \delta z$, and applying the same reasoning in the remaining two directions, we obtain

and

$$
\left.\begin{array}{l}
\frac{d u}{d t}=-\frac{1}{\rho} \cdot \frac{\partial p}{\partial x}-\frac{\partial \Omega}{\partial x}, \\
\frac{d v}{d t}=-\frac{1}{\rho} \frac{\partial p}{\partial y}-\frac{\partial \Omega}{\partial y},
\end{array}\right\}
$$

$$
\frac{d w}{d t}=-\frac{1}{\rho} \frac{\partial p}{\partial z}-\frac{\partial \Omega}{\partial z}
$$

To introduce the local variations of the velocity components, we consider $u, v, w$ as functions of $x, y, z$, and $t$. With this understanding we write the identity

$$
\frac{d u}{d t}=\frac{\partial u}{\partial x} \frac{d x}{d t}+\frac{\partial u}{\partial y} \frac{d y}{d t}+\frac{\partial w}{\partial z} \frac{d z}{d t}+\frac{\partial u}{\partial t},
$$

where $d x / d t$, for example, represents the time variation of the position $x$ of the liquid parallelepiped; that is, the velocity component $u$, and 
$\partial u / \partial t$ represents the time variation of $u$ at the fixed point $(x, y, z)$. Interpreting the quantities $d y / d t$ and $d z / d t$ in the same manner, we write from eq 5 :

Similarly,

$$
\frac{d u}{d t}=\frac{\partial u}{\partial t}+u \frac{\partial u}{\partial x}+v \frac{\partial u}{\partial y}+w \frac{\partial u}{\partial z} .
$$

and

$$
\frac{d v}{d t}=\frac{\partial v}{\partial t}+u \frac{\partial v}{\partial x}+v \frac{\partial v}{\partial y}+w \frac{\partial v}{\partial z}
$$

$$
\frac{d w}{d t}=\frac{\partial w}{\partial t}+u \frac{\partial w}{\partial x}+u \frac{\partial w}{\partial y}+w \frac{\partial w}{\partial z} .
$$

It should be noted that $d u / d t$ represents the acceleration of a particular element of the fluid followed in its motion relative to the axes $x, y, z$. The symbol $d / d t$ has in general this significance.

\section{DISPLACEMENT AND DEFORMATION OF A LIQUID ELEMENT}

We consider the same elementary parallelepiped, $\delta x \delta y \delta z$, having its corner nearest the origin at the point $(x, y, z)$. Designating the component velocities at some instant at the point $(x, y, z)$ by $u, v, w$, we find for the relative velocities at the point $(x+\delta x, y+\delta y, z+\delta z)$ at the same instant, by Taylor's theorem,

and

$$
\left.\begin{array}{c}
\delta u=\frac{\partial u}{\partial x} \delta x+\frac{\partial u}{\partial y} \delta y+\frac{\partial u}{\partial z} \delta z, \\
\delta v=\frac{\partial v}{\partial x} \delta x+\frac{\partial v}{\partial y} \delta y+\frac{\partial v}{\partial z} \delta z,
\end{array}\right\}
$$

If we write

$$
\delta w=\frac{\partial w}{\partial x} \delta x+\frac{\partial w}{\partial y} \delta y+\frac{\partial w}{\partial z} \delta z \cdot
$$

$$
\left.\begin{array}{l}
\epsilon_{1}=\frac{\partial u}{\partial x}, \epsilon_{2}=\frac{\partial v}{\partial y}, \epsilon_{3}=\frac{\partial w}{\partial z}, \\
\gamma_{1}=\frac{\partial w}{\partial y}+\frac{\partial v}{\partial z}, \gamma_{2}=\frac{\partial u}{\partial z}+\frac{\partial w}{\partial x}, \gamma_{3}=\frac{\partial v}{\partial x}+\frac{\partial u}{\partial y},
\end{array}\right\}
$$

and

$$
\xi=\frac{\partial w}{\partial y}-\frac{\partial v}{\partial z}, \eta=\frac{\partial u}{\partial z}-\frac{\partial w}{\partial x}, \zeta=\frac{\partial v}{\partial x}-\frac{\partial u}{\partial y},
$$

the relative velocities may be written

and

$$
\left.\begin{array}{l}
\delta u=\epsilon_{1} \delta x+\frac{1}{2}\left(\gamma_{3} \delta y+\gamma_{2} \delta z\right)+\frac{1}{2}(\eta \delta z-\zeta \delta y), \\
\delta v=\epsilon_{2} \delta y+\frac{1}{2}\left(\gamma_{1} \delta z+\gamma_{3} \delta x\right)+\frac{1}{2}(\zeta \delta x-\xi \delta z), \\
\delta w=\epsilon_{3} \delta z+\frac{1}{2}\left(\gamma_{2} \delta x+\gamma_{1} \delta y\right)+\frac{1}{2}(\xi \delta y-\eta \delta x) \cdot
\end{array}\right\}
$$


The following physical interpretation is now evident. The motion of an elementary parallepiped having the point $(x, y, z)$ for its corner nearest the origin may be considered as made up of three parts. The first part represents a motion of translation of the whole element with the component velocities $u, v, w$. The second part represents the relative displacement of the particles within the parallelepiped and describes its deformation. The linear dilatations $\epsilon_{1}, \epsilon_{2}, \epsilon_{3}$ give the rate of increase of the edges of the element $\delta x, \delta y, \delta z$, respectively, during the motion. The angular dilatations $\gamma_{1}, \gamma_{2}, \gamma_{3}$ give the rate of change of the angles of the edges of the parallepiped, taken two at a time. The third part represents a rotation of the whole element about an instantaneous axis having as components the angular velocities $(1 / 2) \xi,(1 / 2) \eta,(1 / 2) \xi$. The vector $(\xi, \eta, \zeta)$ is referred to as the vorticity of the liquid at the point $(x, y, z)$.

When, throughout a finite portion of a fluid mass, the component vorticities $\xi, \eta, \zeta$ vanish, the motion of that portion is called irrotational.

\section{KINEMATICAL RELATIONS}

Since $\epsilon_{1}, \epsilon_{2}, \epsilon_{3}$ are the rates of dilation of the edges of a cubical element, it can be readily shown that the change, $d V$, of the volume of the liquid mass, $\Delta V=\delta x \delta y \delta z$, due to the displacement of the particles per unit time is

$$
d V=\left(\epsilon_{1}+\epsilon_{2}+\epsilon_{3}\right) V .
$$

We also have the relation,

$$
\rho_{0} V=\left(\rho_{\theta}+\Delta \rho\right)(V+d V),
$$

derived from the principle of conservation of mass. $\rho_{0}$ is the initial density of liquid and $\Delta \rho$ the change in the density during the motion of the element. If the liquid is incompressible, $\Delta \rho=0$, and we obtain from eq 11, $d V=0$, and from eq 10

$$
\frac{\partial u}{\partial x}+\frac{\partial v}{\partial y}+\frac{\partial w}{\partial z}=0
$$

which is a kinematical relation that is satisfied in the region occupied by an incompressible liquid. Equation 12 is known as the equation of continuity for an incompressible liquid.

At the boundary of the liquid the equation of continuity is replaced by a different relation. We shall consider the relation in its most general form. Let $F(x, y, z, t)=0$ be the equation of the bounding surface. Let $u, v, w$ be the velocity components of a liquid particle on the boundary, having the position $(x, y, z)$ at time $t$. At time $t+\Delta t$ the position of the particle is

$$
(x+u \Delta t, y+v \Delta t, z+w \Delta t) .
$$

Since the particle does not leave the boundary [4, p. 7], we must have, because of the equation of the boundary,

$$
F(x+u \Delta t, y+v \Delta t, z+w \Delta t, t+\Delta t)=0 .
$$

When the latter expression is expanded by means of Taylor's theorem, we obtain the relation

$$
\frac{d F}{d t}=\frac{\partial F}{\partial t}+u \frac{\partial F}{\partial x}+v \frac{\partial F}{\partial y}+w \frac{\partial F}{\partial z}=0
$$

which is the general boundary condition. 
Two special forms are to be noted. At a fixed boundary

$$
l u+m v+n w=0,
$$

where $l, m, n$ represent the direction cosines of the normal to the boundary. At a surface of discontinuity

$$
l\left(u_{1}-u_{2}\right)+m\left(v_{1}-v_{2}\right)+n\left(w_{1}-w_{2}\right)=0,
$$

where the suffixes are used to distinguish the velocities at the two sides of the surface.

\section{IRROTATIONAL MOTION}

In a large and important class of hydrodynamical phenomena, the vorticities $\xi, \eta, \zeta$ vanish-that is:

and

$$
\left.\begin{array}{l}
\xi=\frac{\partial w}{\partial y}-\frac{\partial v}{\partial z}=0, \\
\eta=\frac{\partial u}{\partial z}-\frac{\partial w}{\partial x}=0, \\
\zeta=\frac{\partial v}{\partial x}-\frac{\partial u}{\partial y}=0 .
\end{array}\right\}
$$

As a result of this restriction, the expressions for the velocity components, $u, v, w$, are very much simplified.

We consider now the function $\phi$, defined by the differential equation

$$
-d \phi=u d x+v d y+w d z \text {. }
$$

According to a theorem in differential equations, the necessary and sufficient conditions for $d \phi$ to be an exact differential, that is, for $d \phi$ to be expressed as

$$
d \phi=\frac{\partial \phi}{\partial x} d x+\frac{\partial \phi}{\partial y} d y+\frac{\partial \phi}{\partial z} d z
$$

are the relations in eq 16 . Comparing eq 18 with eq 17 , we write for the velocity components of a liquid particle of zero vorticity:

and

$$
\begin{aligned}
& u=-\frac{\partial \phi}{\partial x}, \\
& v=-\frac{\partial \phi}{\partial y},
\end{aligned}
$$

$$
w=-\frac{\partial \phi}{\partial z}
$$

The function $\phi$ is called a velocity potential from its analogy with potentials arising in some other branches of physics. As the velocity potential exists if and only if the flow is irrotational, the latter flow is also called potential flow.

If the liquid, in addition to being in irrotational motion, is also incompressible, then the velocity potential $\phi$ satisfies Laplace's equation,

$$
\frac{\partial^{2} \phi}{\partial x^{2}}+\frac{\partial^{2} \phi}{\partial y^{2}}+\frac{\partial^{2} \phi}{\partial z^{2}}=0
$$


This can be seen by substituting the expressions for the velocities given in eq 19 in the equation of continuity, eq 12 . Thus, for the conditions of flow assumed here, (the irrotational motion of an incompressible liquid) the complete determination of the velocities at all points reduces to the selection of the harmonic function $\phi$ satisfying the boundary conditions.

\section{INVARIANCE OF THE CIRCULATION IN A MOVING CIRCUIT}

The following theorem on irrotational motion is of great importance: If the motion of any portion of a perfect fluid is irrotational at any one moment, it will continue to be irrotational, provided the external forces have a potential and the density of the fluid be either constant or a function of pressure only.

One of the methods for establishing the theorem is to consider the circulation around a closed curve of particles and inquire into the variation of the circulation as the particles of the curve move into new positions. By the circulation around the circuit, $C$, is meant the line integral at any time, $t$,

$$
\Gamma=\oint_{c}(u d x+v d y+w d x),
$$

of the velocity vector taken around $C$, a closed path of particles in the liquid. The circulation of the circuit of the same particles at time $t+d t$ is

where

$$
\Gamma+\frac{d \Gamma}{d t} d t
$$

$$
\frac{d \Gamma}{d t}=\oint_{c} \frac{d}{d t}(u d x+v d y+w d z) .
$$

To evaluate $d \Gamma / d t$ we take an element of circuit $C$ of length $\delta s$ and consider the time-rate of variation of the quantity $u \delta x$ as the particles on the element move into new positions. Differentiating the product $u \delta x$, we obtain

$$
\frac{d}{d t}(u \delta x)=\frac{d u}{d t} \delta x+u \frac{d}{d t} \delta x .
$$

The first term in the right-hand member contains the $x$ component of the acceleration of a particle on $d s$, given by the first of the following dynamical equations of motion

and

$$
\left.\begin{array}{l}
\frac{d u}{d t}=-\frac{1}{\rho} \frac{\partial p}{\partial x}-\frac{\partial \Omega}{\partial x}, \\
\frac{d v}{d t}=-\frac{1}{\rho} \frac{\partial p}{\partial y}-\frac{\partial \Omega}{\partial y},
\end{array}\right\}
$$

$$
\frac{d w}{d t}=-\frac{1}{\rho} \frac{\partial p}{\partial z}-\frac{\partial \Omega}{\partial z} .
$$


Here $p$ is the pressure, $\rho$ the density of liquid, and $\Omega$ the potential of the external forces at the point under consideration. The second term is readily transformed into

$$
u \frac{d}{d t}(\delta x)=u \delta \frac{d x}{d t}=u \delta u,
$$

since $d x / d t$ is the velocity $u$. Thus,

and similarly

$$
\frac{d}{d t}(u \delta x)=-\frac{1}{\rho} \frac{\partial p}{\partial x} \delta x-\frac{\partial \Omega}{\partial x} \delta x+u \delta u,
$$

and

$$
\frac{d}{d t}(v \delta y)=-\frac{1}{\rho} \frac{\partial p}{\partial y} \delta y-\frac{\partial \Omega}{\partial y} \delta y+v \delta v
$$

$$
\frac{d}{d t}(w \delta z)=-\frac{1}{\rho} \frac{\partial p}{\partial z} \delta z-\frac{\partial \Omega}{\partial z} \delta z+w \delta w .
$$

Introducing eq 26 into eq 22 , we obtain

$$
\frac{d \Gamma}{d t}=-\oint_{c} \frac{d p}{\rho}-\oint_{c} d \Omega+\frac{1}{2} \oint_{c} d q^{2}
$$

where $q^{2}=u^{2}+v^{2}+w^{2}$. Since $\Omega$ and $q^{2}$ are single-valued, continuous functions of $x, y, z$, the last two integrals in the right-hand member of the equation vanish. If $\rho$ is a single-valued, nonvanishing, continuous function of $p$ only, or a constant, then the first integral vanishes also. Hence, under these circumstances

$$
\frac{d \Gamma}{d t}=0
$$

that is, the circulation around a circuit moving with the liquid remains unaltered.

By using Stokes' theorem, the circulation, $\Gamma$, around circuit $C$, may be related to the vorticity of the liquid particles on a surface $S$ bounded by $C$. Stokes' theorem states that the line integral of any vector around a closed curve is equivalent to the flux of the curl of the vector through any surface bounded by the closed curve. Using this theorem, we now write

$$
\Gamma=\mathcal{S}_{s}(l \xi+m \eta+n \zeta) d S,
$$

where $l, m, n$ are the direction cosines of the normal drawn inward from the surface.

It now follows from eq 28 and 29 that the property of irrotationality of a portion of the fluid is carried with the fluid. For, if initially $\xi, \eta, \zeta$ vanish in a region containing a fluid, then the circulation for any circuit in the same region vanishes. This is true at all subsequent times in the region containing the same particles, by eq 28 above, and the vorticities consequently remain equal to zero. 
The restrictions under which the main theorem, expressed by eq 28, has been proven must be remembered. It is assumed that the external body forces have a potential, and that the density is either constant or a function of pressure only. The latter condition is violated, for example, in the case of convection currents generated by a temperature gradient. Since in the dynamical eq 24 , which were employed in the proof, the viscosity terms are missing, another restriction is the condition that the effects of viscous forces are negligible in comparison with inertial forces.

\section{IMPULSIVE GENERATION OF MOTION}

When the boundary conditions of a mass of liquid are suddenly changed, a sudden alteration in the motion may take place. The forces introduced are of the nature of impulsive pressures, and, during the time interval that the impulses last, the effects of all the other forces are negligible.

Let $\rho$ be the density, $u_{0}, v_{0}, w_{0}$ the components of velocity immediately before, $u^{\prime}, v^{\prime}, w^{\prime}$ those immediately after the application of the impulse, $\bar{\omega}$ the impulsive pressure at the point $(x, y, z)$. The change of momentum parallel to $x$ of an elementary rectangular parallelepiped having edges $\delta x, \delta y, \delta z$, and with its corner nearest the origin at $(x, y, z)$, is $\rho \delta x \delta y \delta z\left(u^{\prime}-u_{0}\right)$; the resultant impulsive pressure in the

same direction is $-\frac{\partial \bar{\omega}}{\partial x} \delta x \delta y \delta z$. Hence,

$$
\rho \delta x \delta y \delta z\left(u^{\prime}-u_{0}\right)=-\frac{\partial \bar{\omega}}{\partial x} \delta x \delta y \delta z,
$$

or

Similarly

$$
u^{\prime}-u_{0}=-\frac{1}{\rho} \frac{\partial \bar{\omega}}{\partial x} \cdot
$$

and

$$
v^{\prime}-v_{0}=-\frac{1}{\rho} \frac{\partial \bar{\omega}}{\partial y},
$$

$$
w^{\prime}-w_{0}=-\frac{1}{\rho} \frac{\partial \bar{\omega}}{\partial z} .
$$

In these relations we have a physical interpretation of the velocity potential, $\phi$. For, consider the case where $u_{0}, v_{0}, w_{0}$ are zero. A state of motion for which a velocity potential exists could be produced from rest by the application of a properly chosen system of impulsive pressures. If $\phi$ be the velocity potential, the desired system of impulsive pressures is $\bar{\omega}=\rho \phi+C$. The arbitrary constant in this expression shows that a uniform pressure throughout a liquid mass has no effect on the motion. If the initial state of motion be uniform, that is, if for the initial state of motion the liquid possesses the velocity potential $\phi_{0}=u_{0} x+v_{0} y+w_{0} z$, then the new state of motion possessing the velocity potential $\phi$ may be produced from the initial motion by the application of the impulsive pressures $\bar{\omega}=\rho\left(\phi-\phi_{0}\right)+C$. 


\section{PRESSURE EQUATION}

In a case of irrotational motion the velocity potential, $\phi$, determines the velocity components $u, v, w$, but the hydrodynamical solution of the flow is not complete as long as the pressures are unknown. The determination of the pressures is made by means of the pressure equation, which is a dynamic relation to be derived presently.

Since the motion is irrotational, the velocities $u, v, w$ in the dynamical equations,

and

$$
\left.\begin{array}{l}
\frac{d u}{d t}=-\frac{1}{\rho} \frac{\partial p}{\partial x}-\frac{\partial \Omega}{\partial x} \\
\frac{d v}{d t}=-\frac{1}{\rho} \frac{\partial p}{\partial y}-\frac{\partial \Omega}{\partial y},
\end{array}\right\}
$$

$$
\frac{d w}{d t}=-\frac{1}{\rho} \frac{\partial p}{\partial z}-\frac{\partial \Omega}{\partial z},
$$

where the operator $d / d t$ is

$$
\frac{d}{d t}=\frac{\partial}{\partial t}+u \frac{\partial}{\partial x}+v \frac{\partial}{\partial y}+w \frac{\partial}{\partial z},
$$

may be expressed in terms of the velocity potential, $\phi$. The dynamical equations are transformed into

and

$$
\left.\begin{array}{l}
-\frac{\partial^{2} \phi}{\partial x \partial t}+\frac{1}{2} \frac{\partial}{\partial x} q^{2}=-\frac{\partial \Omega}{\partial x}-\frac{1}{\rho} \frac{\partial p}{\partial x}, \\
-\frac{\partial^{2} \phi}{\partial y \partial t}+\frac{1}{2} \frac{\partial}{\partial y} q^{2}=-\frac{\partial \Omega}{\partial y}-\frac{1}{\rho} \frac{\partial p}{\partial y}, \\
-\frac{\partial^{2} \phi}{\partial z \partial t}+\frac{1}{2} \frac{\partial}{\partial z} q^{2}=-\frac{\partial \Omega}{\partial z}-\frac{1}{\rho} \frac{\partial p}{\partial z} .
\end{array}\right\}
$$

Multiplying the first by $d x$, the second by $d y$, the third by $d z$, adding, and then integrating the sum, we obtain the integral

$$
\int \frac{d p}{\rho}=\frac{\partial \phi}{\partial t}-\Omega-(1 / 2) q^{2}+f(t),
$$

where $f(t)$ is an arbitrary function of time. This equation makes it possible to determine the pressure, once the velocity potential $\phi$ is known; it is called the pressure equation.

For an incompressible liquid,

$$
\underline{p}=\frac{\partial \phi}{\partial t}-\Omega-\frac{1}{2} q^{2}+f(t) .
$$

To determine the arbitrary function $f(t)$ we must know $p$ and $\phi$ as functions of $t$ at some definite point in the region occupied by the liquid. Generally this point is one where $\partial \phi / \partial t$ vanishes, $q$ is a constant, and $p$ is the atmospheric pressure $p_{a}$. 


\section{SOLUTION OF PROBLEMS IN IRROTATIONAL MOTION}

The mathematical procedure for solving a problem of the irrotational motion of an incompressible liquid bounded in part by fixed boundaries and in part by a free surface consists in finding a harmonic function, $\phi(x, y, z, t)$, which satisfies Laplace's equation (eq 20) in the region occupied by the liquid, and the following surface conditions.

At the free surface, if it exists, there are two conditions to be satisfied. If $F(x, y, z, t)$ is the equation of the surface, we have

$$
\frac{\partial F}{\partial t}+u \frac{\partial F}{\partial x}+v \frac{\partial F}{\partial y}+w \frac{\partial F}{\partial z}=0,
$$

where $u, v, w$ are the velocities at the surface. The second condition states that the pressure is atmospheric at the free surface; we then have from eq 34

$$
p_{a} / \rho=\frac{\partial \phi}{\partial t}-\Omega-\frac{1}{2} q^{2}+f(t) .
$$

At the fixed boundaries,

$$
\frac{\partial \phi}{\partial n}=0,
$$

$n$ being the normal drawn to the boundary.

The function, $\phi$, which satisfies eq $13,20,35$, and 36 is unique, except for an ladditive constant. For references to proofs see [4, p. 59].

\section{FORMULATION OF THE WAVE PROBLEM-WAVES IN WATER OF SMALL DEPTH}

We consider a rectangular horizontal channel of uniform crosssection and infinite length, containing a perfect liquid initially at rest. We assume that some external agency acting for a short time produces a deformation of the free surface of the liquid in the channel. If the action of the external agency is such that the associated forces exerted on a portion of the water in the channel are essentially in the direction parallel to the direction of the channel and are constant throughout the whole depth of the liquid, the particles in a normal section under the deformed surface will be moving with a constant velocity. Under these conditions the deformed free surface constitutes a translation wave.

The liquid of the channel being initially at rest, we infer from the theorems previously explained that the resulting motion of the liquid under the deformed free surface is irrotational and possesses a velocity potential, $\phi$. Let the axis of $x$ be drawn in the bottom plane of the channel parallel to the channel axis; let the axis of $z$ be drawn upwards. Denoting the velocity components in the direction of $x$ and $z$ by $u$ and $w$, respectively, we have 


$$
u=-\frac{\partial \phi}{\partial x}
$$

and

$$
w=-\frac{\partial \phi}{\partial z}
$$

where $\phi$ is a function of $x, z$, and $t$ and satisfies Laplace's equation

$$
\frac{\partial^{2} \phi}{\partial x^{2}}+\frac{\partial^{2} \phi}{\partial z^{2}}=0 \text {. }
$$

The pressure equation (35) reduces to

$$
\frac{p}{\rho}=\frac{\partial \phi}{\partial t}-g z-\frac{1}{2}\left(u^{2}+w^{2}\right)+F(t),
$$

where $g$ is the intensity of gravity.

Let the $z$ coordinate of the free surface be denoted by $h+H$, where $H$ is the initial undisturbed depth of water in the channel, and $h$ is the elevation (or depression) of the wave.

To evaluate $F(t)$, let us consider the point at infinity on the free surface; at such a point $\partial \phi / \partial t=u=w=h=0, z=H$, and $p=p_{a}$. Substituting in eq 40 there results

$$
F(t)=\frac{p_{a}}{\rho}+g H .
$$

At the surface, since $p=p_{a}$, we obtain the dynamical surface condition from eq 40, neglecting the effect of surface tension:

$$
\frac{\partial \phi}{\partial t}-g h-\frac{1}{2}\left(u^{2}+w^{2}\right)=0, z=H+h .
$$

Although the last equation has been derived for the case where the liquid at infinity is at rest, it is equally applicable to the case where $u^{2}+w^{2}$ and $\partial \phi / \partial t$ vanish at the points on the surface where $h=0$, as, for example, in an intumescence consisting of an infinite number of undulations of the same dimensions moving without change of form.

We obtain the kinematic surface condition, that a fluid particle of the free surface always remains on the free surface, by substituting $F(x, y, z, t)=z-H-h=0$ in eq 13 , obtaining

$$
w=\frac{\partial h}{\partial t}+u \frac{\partial h}{\partial x}, z=H+h .
$$

At the bottom surface of the channel the velocity component normal to the bottom vanishes, that is, at $z=0$,

$$
\frac{\partial \phi}{\partial z}=0 .
$$

In view of this last condition, we adopt for the potential $\phi$ the power series

$$
\phi=\sum_{n=0}^{\infty} \phi_{n} z^{n}, \phi_{1}=0,
$$


where $\phi_{n}$ is a function of $x$ and $t$ only. Substituting this expression for $\phi$ in eq 39, and equating the coefficients of $z^{n}$ to zero, term by term, we arrive at the convergent series

$$
\phi=\phi_{0}-\frac{z^{2}}{2 !} \frac{\partial^{2} \phi_{0}}{\partial x^{2}}+\frac{z^{4}}{4 !} \frac{\partial^{4} \phi_{0}}{\partial x^{4}}-\frac{z^{6}}{6 !} \frac{\partial^{6} \phi_{0}}{\partial x^{6}}+\ldots .
$$

Differentiating with respect to $z$,

$$
\frac{\partial \phi}{\partial z}=-z \frac{\partial^{2} \phi_{0}}{\partial x^{2}}+\frac{z^{3}}{3 !} \frac{\partial^{4} \phi_{0}}{\partial x^{4}}-\frac{z^{5}}{5 !} \frac{\partial^{6} \phi_{0}}{\partial x^{6}}+\ldots .
$$

The function $\phi_{0}$, as stated above, depends only on $x$ and $t$. If we denote the velocity of particles of water at the bottom of the channel at $x$ by $u_{0}$, then

$$
\phi_{0}=\left(\phi_{0}\right)_{\infty}-\int_{x}^{\infty} \frac{\partial \phi_{0}}{\partial x} d x=\left(\phi_{0}\right)_{\infty}+\int_{x}^{\infty} u_{0} d x,
$$

where $\left(\phi_{0}\right)_{\infty}$ is the value of $\phi$ at $x=\infty$. Since the liquid in the channel at infinity is at rest, we may take the value of $\left(\phi_{0}\right)_{\infty}$ as zero: then,

$$
\phi_{0}=\int_{x}^{\infty} u_{0} d x .
$$

In the above formulation, $h$ and $\phi_{0}$ are functions specifying a translation wave. They can be determined by integrating the two simultaneous differential eq 41 and 42 representing the dynamical and kinematical surface conditions.

\section{LONG WAVES OF NEGLIGIBLE HEIGHT AND CURVATURE}

\section{VELOCITY OF PROPAGATION}

If $u^{2}$ and $w^{2}$ are negligible in comparison with gh (assumptions I, II) and $u \partial h / \partial x$ in comparison with $\partial h / \partial t$ (assumption III), then the surface conditions given by eq 41 and 42 reduce to

$$
g h-\frac{\partial \phi}{\partial t}=0,
$$

and

$$
\frac{\partial \phi}{\partial z}+\frac{\partial h}{\partial t}=0
$$

Also, if in the expansions of $\phi$ and $\partial \phi / \partial z$ given in eq 45 and 46 , only the first term is retained (assumption IV), we have

$$
\phi=\phi_{0},
$$

and

$$
\frac{\partial \phi}{\partial z}=-z \frac{\partial^{2} \phi_{0}}{\partial x^{2}} .
$$

$198881-40-5$ 
Here, then, we have the basis for a first approximation to a solution of a wave configuration. The horizontal component of the velocity in a normal section is constant and equals the bottom velocity $u_{0}$; the vertical component varies linearly with $z$.

Substituting eq 51 and 52 in eq 49 and 50, we obtain

and

$$
g h-\frac{\partial \phi_{0}}{\partial t}=0,
$$

$$
-H \frac{\partial^{2} \phi_{0}}{\partial x^{2}}+\frac{\partial h}{\partial t}=0
$$

assuming that $h$ is small in comparison with $H$ and hence can be neglected (assumption V). Eliminating $\phi_{0}$ from eq 53 and 54, we obtain the differential equation

where

$$
\frac{\partial^{2} h}{\partial t^{2}}=c^{2} \frac{\partial^{2} h}{\partial x^{2}},
$$

$$
c=\sqrt{g H} .
$$

Eliminating $h$ similarly and writing

$$
u_{0}=-\frac{\partial \phi_{0}}{\partial x},
$$

there results for $u_{0}$ the relation

$$
\frac{\partial^{2} u_{0}}{\partial t^{2}}=c^{2} \frac{\partial^{2} u_{0}}{\partial x^{2}}
$$

Equations 55 and 58, which are well known, possess complete solutions, which also satisfy eq 53 and 54:

and

$$
\frac{h}{H}=F_{1}(x-c t)+F_{2}(x+c t),
$$

$$
\frac{u_{0}}{c}=F_{1}(x-c \hat{t})-F_{2}(x+c t),
$$

where $F_{1}$ and $F_{2}$ are two arbitrary functions.

These results have a very simple geometrical interpretation. Consider the motion represented by the function $F_{1}$ in eq 60 . When $x$ and $t$ are increased by $c \tau$ and $\tau$, respectively, the value of the function is not changed; the disturbance $h$ which existed at the place $x$ and at time $t$ has moved during the time interval $\tau$ to the place $x+c \tau$. Therefore, the function $F_{1}$ represents a wave traveling in the positive $x$-direction without change of form at a constant velocity of propagation ${ }^{2} c=\sqrt{g H}$.

Similarly, $F_{2}$ represents another wave propagated in the opposite direction with the same velocity. Any motion whatever subject to the conditions assumed above for the approximate solution will be made up of waves of these types. $F_{1}$ and $F_{2}$ are determinate when

\footnotetext{
3 The question of the meaning of velocity of propagation when the wave does not retain its form will be discussed later in section $\mathrm{V}-1$.
} 
$h$ and $u_{0}$ are known for every value of $t$, and at a single point $x=x_{1}$; or when $h$ and $u_{0}$ are known for every value of $x$ and for a single time $t=t_{1}$.

If $h$ and $u_{0}$ represent the surface elevation and velocity of particles in either the progressive or regressive wave $\left(F_{1}\right.$ or $\left.F_{2} \equiv 0\right)$ it is seen from eq 59 and 60 that

$$
u_{0}= \pm c \frac{h}{\bar{H}},
$$

where the plus sign applies to a wave moving in the positive $x$-direction $\left(F_{2} \equiv 0\right)$ and the minus sign to a wave moving in the negative $x$-direction $\left(F_{1} \equiv 0\right)$.

We shall now examine the restrictions assumed in obtaining our approximate solution:

and

$$
\begin{aligned}
& u^{2} \ll g h, \\
& w^{2} \ll g h,
\end{aligned}
$$

$$
u \frac{\partial h}{\partial x} \ll \frac{\partial h}{\partial t} .
$$

The two series converge rapidly; that is,

$$
\frac{z^{2}}{2 !} \frac{\partial^{2} \phi_{0}}{\partial x^{2}} \ll \phi_{0},
$$

and

$$
\frac{h}{H} \ll 1 .
$$

It can be demonstrated that the following physical restrictions lead to the above mathematical assumptions:

(A) The ratio of the wave amplitude to the depth of the channel is small: $(h / H) \ll 1$.

(B) The slope of the wave is small: $(\partial h / \partial x) \ll 1$.

(C) The radius of curvature of the waves is large with respect to $H^{2} / 2 h$.

\section{ENERGY OF AN INTUMESCENCE}

A single wave is called an intumescence. The potential energy per unit width of an intumescence, at time $t$, due to the elevation or depression of the fluid surface above or below the level of the previously undisturbed liquid in a channel is

$$
\frac{g \rho}{2} \int_{x_{1}}^{x_{2}} h^{2} d x
$$

where $x_{1}$ and $x_{2}$ denote the extremities of the intumescence. The kinetic energy per unit width of the liquid under the free surface of the intumescence is

$$
\frac{\rho H}{2} \int_{x^{1}}^{x_{1}} u_{0}^{2} d x
$$


Substituting for $u_{0}$ its value in eq 61 , eq 63 becomes

$$
\frac{\rho g}{2} \int_{x_{1}}^{x_{2}} h^{2} d x .
$$

Comparing eq 64 and 62 , it is seen that the potential and kinetic energy of an intumescence are equal and that the total energy is

$$
g \rho \int_{x_{1}}^{x_{2}} h^{2} d x .
$$

The integral in eq 65 without the multiplier $g \rho$ will be of special interest, and will be subsequently referred to as the energy, $E$, of an intumescence.

\section{DISPLACEMENT OF THE PARTICLES}

The liquid particles in the cross section at $x$, before the arrival of an intumescence, are at rest. At the moment when the intumescence is passing, the particles in the section are moving with the velocity $u_{0}$, to a first approximation. Thus, the volume of flow through the section during the passage of the intumescence is

$$
V=\int_{t_{1}}^{t_{2}} u_{0} H d t
$$

Since

$$
c d t=d x
$$

we have

$$
V=\int_{x_{1}}^{x_{2}} \frac{u_{0} H}{C} d x .
$$

Substituting for $u_{0}$ from eq 61 , we obtain

$$
V=\int_{x_{1}}^{x_{2}} h d x .
$$

The volume of flow through a cross section equals the volume of the intumescence. Hence, during the passage of an intumescence, the particles in a cross section are moved forward a distance $\delta x$, the magnitude of which is given by the ratio of the volume of the intumescence to the area of the channel cross section.

\section{EFFECT OF AN ARBITRARY INITIAL DISTURBANCE}

If for $t=0$ the velocity of the liquid particles in the channel and the form of the disturbed surface are known:

$$
\frac{u}{c}=\theta(x),-\infty<x<+\infty,
$$

and

$$
\frac{h}{H}=\psi(x),-\infty<x<+\infty,
$$


then the functions $F_{1}$ and $F_{2}$ occurring in eq 59 and 60 are

and

$$
\left.\begin{array}{c}
F_{1}(x)=\frac{1}{2}[\theta(x)+\psi(x)], \\
F_{2}(x)=\frac{1}{2}[-\theta(x)+\psi(x)] .
\end{array}\right\}
$$

To determine the form of the wave profile, $h / H=\psi\left(x, t_{1}\right)$ for any subsequent time, $t=t_{1}$, the curve representing $F_{1}(x)$ is displaced in the positive $x$-direction by a distance $c t_{1}$, the curve representing $F_{2}(x)$ by the same distance in the opposite direction, and the ordinates of the two displaced curves are added algebraically.

Consider disturbances confined initially to a finite segment of the channel of length $l$. Resolution of the initial disturbance into $F_{1}$ and $F_{2}$ indicates that after the lapse of the time interval $\tau=c / 2$, two waves of equal length $l$ will be traveling in opposite directions. In general, resolved waves will have unequal heights at corresponding points. If, however, in the initial state, $u=0$, that is, $\theta(x) \equiv 0$, then $F_{1}(x) \equiv$ $F_{2}(x)$, and the two component waves have the same shape. If, on the other hand, $u= \pm c h / H$ initially, then either $F_{1}$ or $F_{2}$ is zero, and the result is a single wave traveling in one direction.

\section{REFLECTION AND TRANSMISSION OF WAVES}

A wave is reflected when it reaches a barrier in a channel. We take the origin, $x=0$, at the barrier and note that $u=0$ at the barrier. Utilizing this fact in the general eq 60 , we obtain the condition that

$$
F_{1}(-c t)=F_{2}(+c t)
$$

for the incident and reflected waves.

Applying this last condition to the general eq 59 and 60 , we obtain

and

$$
\frac{h}{H}=F_{2}(c t-x)+F_{2}(c t+x),
$$

$$
\frac{u_{0}}{c}=F_{2}(c t-x)-F_{2}(c t+x),
$$

which are the equations for reflected waves or for identical waves traveling in opposite directions. Here, if $F_{2}(c t+x)$ is taken as the incident wave, $F_{2}(c t-x)$ will be the reflected wave. At $x=x_{1}$, and $t=t_{1}$, the elevation or depression of the wave is

$$
\frac{h}{H}=F_{2}\left(c t_{1}+x_{1}\right) .
$$

The corresponding elevation or depression of the reflected wave will reach the point $x=x_{1}$ at the time $t=t_{1}+2 x_{1} / c$, and the value of the elevation or depression is

$$
\frac{h}{H}=F_{2}\left(c\left\{t_{1}+2 x_{1} / c\right\}-x_{1}\right)=F_{2}\left(c t_{1}+x_{1}\right) .
$$


Hence, elevations and depressions are reflected without change. It can be shown in a similar manner that corresponding velocities are reversed with their magnitudes unchanged.

When a wave reaches a point in a channel where there is an abrupt change in the cross section of the channel, either in width or in depth, a part of the approaching wave is transmitted and a part is reflected. Taking the origin at the point of the abrupt change of section, we may write from the general eq 59 and 60 for the side from which the wave approaches, moving in the positive $x$-direction,

$$
\frac{h_{1}}{H_{1}}=F\left(x-c_{1} t\right)+f\left(c_{1} t+x\right),
$$

and

$$
\frac{u_{1}}{c_{1}}=F\left(x-c_{1} t\right)-f\left(c_{1} t+x\right),
$$

in which the first term in the right member of each equation applies to the approaching wave, and the second term to the reflected wave.

For the transmitted wave we have

and

$$
\frac{h_{2}}{H_{2}}=g\left(x-c_{2} t\right),
$$

$$
\frac{u_{2}}{c_{2}}=g\left(x-c_{2} t\right) \text {. }
$$

In the above equations $H_{1}$ and $H_{2}$ are the depths of the channel on the two sides of $x=0$, and $c_{1}$ and $c_{2}$ the velocities of propagation for the corresponding portions of the channel. At $x=0, h_{1}=h_{2}$, since the surface is continuous, and therefore,

$$
H_{1}\left[F\left(-c_{1} t\right)+f\left(c_{1} t\right)\right]=H_{2} g\left(-c_{2} t\right) .
$$

Also, the conservation of matter requires that $B_{1} H_{1} u_{1}=B_{2} H_{2} u_{2}$, where $B_{1}$ and $B_{2}$ are the widths of the channel on the two sides of $x=0$. This condition gives

$$
B_{1} H_{1} c_{1}\left[F\left(-c_{1} t\right)-f\left(c_{1} t\right)\right]=B_{2} H_{2} c_{2} g\left(-c_{2} t\right) .
$$

We deduce from eq 83 and 84 the ratio of the elevations in the corresponding parts of the reflected and incident waves and of the transmitted and incident waves. These are

$$
\frac{f}{F}=\frac{B_{1} c_{1}-B_{2} c_{2}}{B_{1} c_{1}+B_{2} c_{2}}
$$

and

$$
\frac{g}{F}=\frac{2 B_{1} c_{1} H_{1}}{\left(B_{1} c_{1}+B_{2} c_{2}\right) H_{2}},
$$

respectively.

A wave traveling in a channel connected to a body of water in a basin of infinite depth and width may be considered as a special case. For this case it is evident by inspection that $g / F=0$ and $f / F=-1$. Hence the incident wave is completely reflected at the juncture, and the elevations are reflected as depressions. 


\section{WAVES OF APPRECIABLE HEIGHT AND CURVATURE}

\section{TWO DEFINITIONS OF VELOCITY OF PROPAGATION}

For long waves of infinitesimal height, the concept of velocity of propagation entails no ambiguity, because such waves are propagated without change of form. However, when a traveling wave is undergoing deformation, the meaning of velocity of propagation must be clearly defined. We may adopt either of two distinct definitions. The first definition will be developed on a purely formal basis. In a given wave system we consider the quantities $\partial h / \partial t$ and $\partial h / \partial x$ for an arbitrary $x$ and $t$, and write the following relation from eq 13:

$$
\frac{\partial h}{\partial t}+\omega_{1} \frac{\partial h}{\partial x}=0, h=h(x, t) .
$$

In effect, this relation defines the quantity $\omega_{1}$, which we may call the velocity of propagation of a wave-height element. When the traveling wave is undergoing a change of form at a given instant, $t, \omega_{1}$ is a function of $h$.

A second definition, which is due to Boussinesq, considers the volume of an intumescence. The volume of an intumescence is the quantity of liquid in the entire intumescence between the free surface and the undisturbed primitive level of the liquid in channel. We have, therefore

$$
\Sigma=\int_{x_{0}}^{\infty} h d x,
$$

where $x_{0}$ is the rear of the intumescence, assumed moving in the positive $x$-direction. The intumescence may be limited posteriorly; that is, for all values of $x \leqq x_{0}, h$ is negligible, and in this case $\Sigma$ has a finite value. On the other hand, the intumescence may be unlimited; in this case we consider the volume, $\Sigma$, included between the head and an abscissa, $x_{0}$, considerably removed from the head, and moving in such a way that $\Sigma$ remains constant.

We now consider a partial volume, $\sigma$, of $\Sigma$, between the head of the intumescence and an abscissa, $x$ :

$$
\sigma=\int_{x}^{\infty} h d x, x \leqq x_{0} .
$$

We assume that a plane originally at $x$ moves in such a way that it always has in front of it the same partial volume $\sigma$ of the intumescence. We refer to the velocity at which this plane moves as the velocity of propagation of an element of volume of an intumescence, and denote this velocity by $\omega$.

The relation between $\omega$ and $h$ may be obtained from eq 88 by differentiating the right-hand member of the equation with respect to $t$ and equating to zero. However, it may be more instructive to obtain the desired relation in the following manner (see fig. 1). The curves $a b c$ and $a^{\prime} b^{\prime} c^{\prime}$ represent the free surface of the moving intumescence at the times $t$ and $t+\Delta t$, respectively. A plane at $x$ having in front of it, at time $t$, the partial volume, $\sigma$, of the intumescence, moves to the position $x+\omega \Delta t$ at time $t+\Delta t$ and still has the same par- 


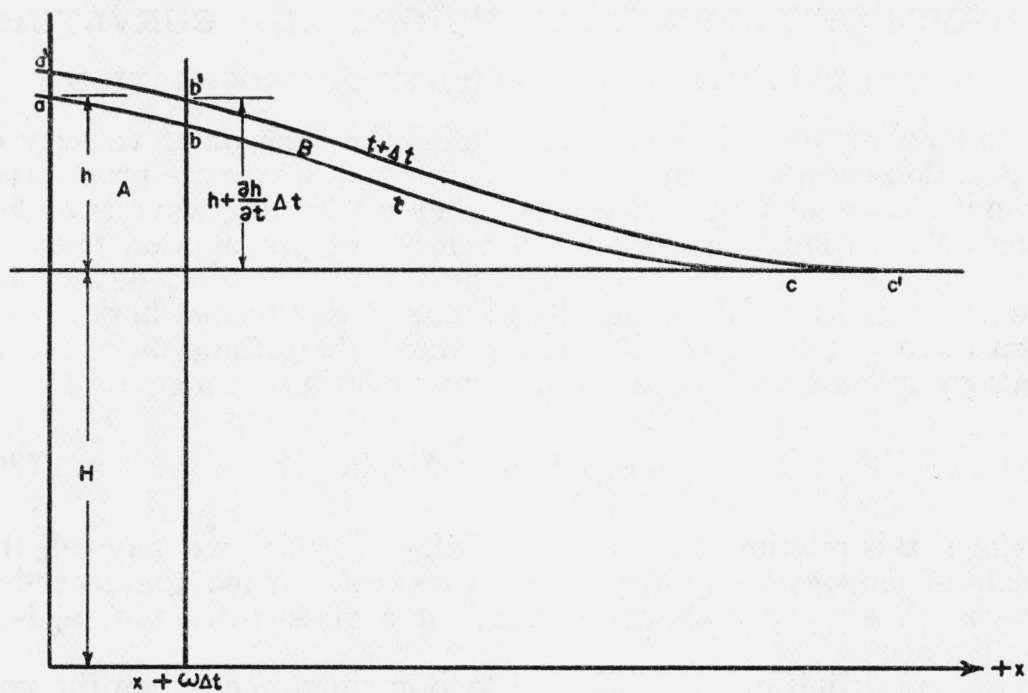

FiguRe 1.-Diagram illustrating the velocity of propagation of a volume element.

tial volume, $\sigma$, in front of it. The areas $A$ and $B$ are equal to each other. Consequently,

Dividing by $\Delta t$,

$$
h \omega \Delta t=\int_{x}^{\infty} \frac{\partial h}{\partial t} \Delta t d x .
$$

$$
h \omega=\int_{x}^{\infty} \frac{\partial h}{\partial t} d x, x \leqq x_{0},
$$

and then differentiating with respect to $x$, we obtain

$$
\frac{\partial h}{\partial t}+\frac{\partial}{\partial x}(h \omega)=0,
$$

which is the relation connecting $\omega$ with $h$.

Eliminating $\partial h / \partial t$ between eq 86 and 90 , we find that the difference between the two velocities of propagation is

$$
\omega_{1}-\omega=h \frac{\partial \omega}{\partial x} / \frac{\partial h}{\partial x} .
$$

If a wave is moving without change of form, $\omega$ is constant, and the two definitions give identical values for the two velocities of propagation, a fact that is self-evident.

\section{MEAN VELOCITY OF PARTICLES}

If $U$ and $q$, respectively, denote the mean velocity of the particles in, and the discharge through, a cross section at $x$, then

$$
q=U(H+h)
$$

Consider the inflow and outflow in a time interval, $\Delta t$, for the volume between two cross sections separated by a distance, $\Delta x ; \Delta x$ 
and $\Delta t$ having the same order of magnitude. The mean discharge at $x$ in the time $\Delta t$ is

$$
q+\frac{\Delta t}{2} \frac{\partial q}{\partial t},
$$

omitting infinitesimals in $\Delta t$ of the second and higher orders. The volume of the inflow in the time $\Delta t$ is then

$$
q \Delta t+\frac{(\Delta t)^{2}}{2} \frac{\partial q}{\partial t}
$$

Similarly, the mean discharge at $x+\Delta x$ in the time $\Delta t$ is

$$
q+\frac{\partial q}{\partial x} \Delta x+\frac{\Delta t}{2} \frac{\partial q}{\partial t}
$$

omitting infinitesimals in $\Delta x$ and $\Delta t$ of the second and higher orders. The volume of the outflow at $x+\Delta x$ in the time $\Delta t$ is

$$
q \Delta t+\frac{\partial q}{\partial x} \quad \Delta x \Delta t+\frac{(\Delta t)^{2}}{2} \frac{\partial q}{\partial t},
$$

and the accumulation of liquid in the volume is

$$
-\frac{\partial q}{\partial x} \Delta x \Delta t \text {. }
$$

The increase of volume of the liquid is also

$$
\frac{\partial h}{\partial t} \Delta x \Delta t,
$$

omitting infinitesimals of the same orders as before.

Therefore,

$$
\frac{\partial q}{\partial x}=-\frac{\partial h}{\partial t}
$$

or from eq 92

$$
\frac{\partial}{\partial x}[U(H+h)]=-\frac{\partial h}{\partial t} .
$$

Substituting from eq 90

$$
\frac{\partial}{\partial x}[U(H+h)]=\frac{\partial}{\partial x}(h \omega),
$$

which when integrated becomes

$$
U(H+h)=h \omega+C .
$$

Since the liquid is assumed to be undisturbed at infinity, the constant of integration vanishes, and the equation for the mean velocity is

$$
U=\frac{h \omega}{H+h} .
$$




\section{EFFECT OF CURVATURE AND HEIGHT ON VELOCITY OF PROPAGATION OF A VOLUME ELEMENT}

When the ratio $h / H$ and the curvature of the wave profile are appreciable, the first approximation is no longer satisfactory, and in such cases recourse to a second approximation becomes necessary. The method which will now be presented is due essentially to Boussinesq.

In the surface conditions, eq 41 and 42 , we neglect $w^{2}$ and replace $u$ by the value of $u_{0}$ in the first approximation, eq 61 , replacing $c$ by its value in eq 56 :

These conditions now become

$$
u=\sqrt{\frac{g}{H}} h .
$$

and

$$
g h-\frac{\partial \phi}{\partial t}+\frac{1}{2} \frac{g}{H} h^{2}=0,
$$

$$
\frac{\partial \phi}{\partial z}+\frac{\partial h}{\partial t}+\sqrt{\frac{g}{H}} h \frac{\partial h}{\partial x}=0 .
$$

Retaining the first two terms in the expressions for $\phi$ and $\partial \phi / \partial z$, eq 45 and 46, and neglecting $h$ in the terms having the smaller values, we obtain

$$
\phi=\phi_{0}-\frac{H^{2}}{2} \frac{\partial^{2} \phi_{0}}{\partial x^{2}}
$$

and

$$
\frac{\partial \phi}{\partial z}=-(h+H) \frac{\partial^{2} \phi_{0}}{\partial x^{2}}+\frac{H^{3}}{6} \frac{\partial^{4} \phi_{0}}{\partial x^{4}}
$$

We differentiate eq 100 with respect to $t$, replacing $\partial \phi_{0} / \partial t$ in the second term of the right-hand member by its value in the first approximation given by eq 53 , that is, by $g h$. In this manner we obtain

$$
\frac{\partial \phi}{\partial t}=\frac{\partial \phi_{0}}{\partial t}-\frac{H^{2}}{2} g \frac{\partial^{2} h}{\partial x^{2}} \text {. }
$$

We next consider eq 101 . In the right-hand member there are two small terms. In these terms we replace $\partial \phi_{0} / \partial x$ by its first approximation from eq 57 and 61 , that is, by $-\sqrt{\frac{g}{H}} h$. In this manner, restricting ourselves to waves propagated in the positive $x$-direction, we obtain

$$
\frac{\partial \phi}{\partial z}=-H \frac{\partial^{2} \phi_{0}}{\partial x^{2}}+\sqrt{\frac{g}{H}} h \frac{\partial h}{\partial x}-\frac{H^{3}}{6} \sqrt{\frac{g}{H}} \frac{\partial^{3} h}{\partial x^{3}} .
$$

Substituting from eq 102 and 103, we obtain from eq 98 and 99:

and

$$
g h-\frac{\partial \phi_{0}}{\partial t}+\frac{g}{2}\left[\frac{h^{2}}{H}+H^{2} \frac{\partial^{2} h}{\partial x^{2}}\right]=0,
$$

$$
\frac{\partial h}{\partial t}-H \frac{\partial^{2} \phi_{0}}{\partial x^{2}}+\sqrt{g H} \frac{\partial}{\partial x}\left[\frac{h^{2}}{H}-\frac{H^{2}}{6} \frac{\partial^{2} h}{\partial x^{2}}\right]=0 .
$$


To eliminate $\phi_{0}$ between these, we differentiate eq 104 twice with respect to $x$ and multiply through by $H$, and differentiate eq 105 with respect to $t$. In place of actually differentiating with respect to $t$, however, we differentiate with respect to $x$ and multiply ${ }^{3}$ by $-\sqrt{g H}$. Subtracting eq 105 from eq 104, there results:

$$
\frac{\partial^{2} h}{\partial t^{2}}=g H \frac{\partial^{2} h}{\partial x^{2}}+g H \frac{\partial^{2}}{\partial x^{2}}\left(\frac{3}{2} \frac{h^{2}}{H}+\frac{H^{2}}{3} \frac{\partial^{2} h}{\partial x^{2}}\right),
$$

which is the fundamental wave equation to the second approximation, in which the effects of the wave height and of curvature of wave profile are included.

Although this equation has been derived for the case where the liquid in the channel is undisturbed at infinity, it remains valid for the case where the liquid at infinity is in motion, provided $u=\sqrt{g / \mathrm{H}} h$, to a first approximation (eq 97).

Equation 106 becomes integrable once with respect to $x$ when $\partial h / \partial t$ is introduced from eq 90. Performing the integration with respect to $x$ and remembering that $h$ and its derivatives vanish for $x=\infty$, we obtain

$$
\frac{\partial}{\partial t}(h \omega)+g H \frac{\partial h}{\partial x}+g H \frac{\partial}{\partial x}\left(\frac{3 h^{2}}{2 H}+\frac{H^{2}}{3} \frac{\partial^{2} h}{\partial x^{2}}\right)=0,
$$

which permits the determination of $\omega$, the velocity of propagation of a wave-volume element, in terms of $h$ and $\partial^{2} h / \partial x^{2}$.

We adopt as an expression for $\omega$ the product

$$
\omega=\sqrt{g \bar{H}}(1+\epsilon),
$$

where $\epsilon$ is a small quantity. Multiplying the two members by $h$ and differentiating with respect to $t$, we obtain, remembering that $\epsilon$ is a small quantity and applying the same reasoning used in differentiating eq 105 ,

$$
\frac{\partial}{\partial t}(h \omega)=\sqrt{g H} \frac{\partial h}{\partial t}-g H \frac{\partial}{\partial x}(\epsilon h) .
$$

Introducing $\partial h / \partial t$ from eq 90 , this"may be written,

$$
\frac{\partial}{\partial t}(h \omega)=-\sqrt{g H} \frac{\partial}{\partial x}(h \omega)-g H \frac{\partial}{\partial x}(\epsilon h) ;
$$

or, in view of eq 108 ,

$$
\frac{\partial}{\partial t}(h \omega)=-g H \frac{\partial}{\partial x}[(1+2 \epsilon) h] .
$$

3 The justification for replacing the operator $\partial / \partial t$ by $-\sqrt{g H} \partial / \partial x$ can be seen from the following: Assuming that the wave retains its form, that is, that $d F / d t=0$, where $F$ is a function of $(x-c t)$, we have the relation

$$
\frac{d F}{d t}-\frac{\partial F}{\partial t}+c \frac{\partial F}{\partial x}=0
$$

from which it is obvious that

$$
\frac{\partial F}{\partial t}=-c \frac{\partial F}{\partial x}=-\sqrt{g H} \frac{\partial F}{\partial x}
$$

While the present discussion relates to waves that may change their form, the above relation can be applied to small terms in the wave equation, to a first approximation. 
The substitution of eq 110 in eq 107 makes the latter expression immediately integrable with respect to $x$. Integrating, putting the constant of integration equal to zero, since $h$ and its derivatives vanish at $x=\infty$, and then dividing by $h$, we find

$$
2 \epsilon=\frac{3}{2} \frac{h}{H}+\frac{H^{2}}{3 h} \frac{\partial^{2} h}{\partial x^{2}} \text {. }
$$

Hence

$$
\omega=\sqrt{g H}\left(1+\frac{3}{4} \frac{h}{H}+\frac{H^{2}}{6 h} \frac{\partial^{2} h}{\partial x^{2}}\right),
$$

which is the expression for the velocity of propagation of a volume element of an intumescence moving in still water.

We next derive the expressions for $u_{0}$, the horizontal velocity component at the bottom, and $u, w$, the velocity components at a point having the ordinate $z$. To determine $u_{0}$ we consider eq 105, replacing $\partial h / \partial t$ by $-\partial(h \omega) / \partial x$ from eq 90 , and then integrating with respect to $x$, the constant of integration being equated to zero. In this manner we obtain, since $u_{0}=-\partial \phi_{0} / \partial x$,

$$
u_{0}=\sqrt{\frac{g}{H}}\left(h-\frac{h^{2}}{4 H}+\frac{H^{2}}{3} \frac{\partial^{2} h}{\partial x^{2}}\right) .
$$

From eq 45, after differentiating with respect to $x$, and from eq 46 , retaining only the first two terms of the right members, we obtain:

and

$$
u=u_{0}-\frac{z^{2}}{2} \frac{\partial^{2} u_{0}}{\partial x^{2}}
$$

$$
w=-z \frac{\partial u_{0}}{\partial x}+\frac{z^{3}}{6} \frac{\partial^{3} u_{0}}{\partial x^{3}}
$$

which give, after substituting for $u_{0}$ from eq 113 , and neglecting quantities of small order:

and

$$
u=\sqrt{g H}\left[\frac{h}{H}-\frac{h^{2}}{4 H^{2}}+\left(\frac{H}{3}-\frac{z^{2}}{2 H}\right) \frac{\partial^{2} h}{\partial x^{2}}\right],
$$

$$
w=-z \sqrt{g H}\left[\left(\frac{1}{H}-\frac{h}{2 H^{2}}\right) \frac{\partial h}{\partial x}+\frac{1}{3}\left(H-\frac{z^{2}}{2 H}\right) \frac{\partial^{3} h}{\partial x^{3}}\right] \text {. }
$$

\section{DEFORMATION OF THE WAVE PROFILE}

The expression for $\omega$, eq 112, and the relation in eq 90 permit the evaluation of the change in $h$ occurring during a short interval of time to a sufficient approximation. Denoting $\sqrt{g H}$ by $\omega_{0}$, eq 90 may be written as

$$
\frac{\partial h}{\partial t}+\omega_{0} \frac{\partial h}{\partial x}+\frac{\partial}{\partial x}\left[h\left(\omega-\omega_{0}\right)\right]
$$

The integral of this expression is

$$
h=f\left(x-\omega_{0} t\right)-t \frac{\partial}{\partial x}\left[h\left(\omega-\omega_{0}\right)\right],
$$


when $t$ is small. This may be proved by differentiating eq 119 and substituting in eq 118 . In the process of differentiating the second term in the right member, the relation $\partial / \partial t=\omega_{0} \partial / \partial x$ is used, this being valid if $t$ is small. Eq 119 enables us to trace the wave profile curve as it would be for time $t_{1}+t$, if it is given for time $t_{1}$.

We next take an element of the intumescence, $d \sigma$, having the height $h$ and base $d x$ and follow its motion. We denote the time-rate of variation of $h$ during the motion by $d h / d t$. From the definition of $\omega$ we obtain

$$
\frac{d h}{d t}=\frac{\partial h}{\partial t}+\omega \frac{\partial h}{\partial x}
$$

Multiplying by $h$ and using eq 90 , we obtain

$$
h \frac{d h}{d t}=-h^{2} \frac{\partial \omega}{\partial x} .
$$

Introducing the value of $\omega$ from eq 112 , we obtain

$$
h \frac{d h}{d t}=-\sqrt{g H}\left[\frac{3 h^{2} \partial h}{4 H \partial x}-\frac{H^{2}}{6} \frac{\partial h}{\partial x} \frac{\partial^{2}}{\partial x^{2}}+\frac{h H^{2}}{6} \frac{\partial^{3} h}{\partial x^{3}}\right],
$$

which may be written, after dividing through by $h$,

$$
\frac{d h}{d t}=-\sqrt{g H} \frac{1}{h} \frac{\partial}{\partial x}\left\{\frac{h^{3}}{4 H}+\frac{H^{2}}{6}\left[h \frac{\partial^{2} h}{\partial x^{2}}-\left(\frac{\partial h}{\partial x}\right)^{2}\right]\right\} .
$$

This expression gives the rate of variation of the height of an intumescence element.

\section{MOTION OF THE CENTER OF GRAVITY OF A WAVE}

The last section treated of the motion of an element, $d \sigma$, of a wave. We may also discuss the motion of a volume, $V$, between two abscissas, $x_{1}$ and $x_{2}$ where $x_{2}>x_{1}$. Then

$$
V=\int_{x_{1}}^{x_{3}} d \sigma
$$

Let $\xi$ denote the horizontal coordinate of the center of gravity of the volume and $\eta$ the elevation of the center of gravity above the level of the undisturbed liquid in the channel. By definition,

$$
\xi=\frac{\int_{x_{1}}^{x_{2}} x d \sigma}{V} \text {, and } \eta=\frac{\int_{x_{1}}^{x_{2}} h d \sigma}{2 V} .
$$

Differentiating with respect to $t$, we obtain

$$
\frac{d \xi}{d t}=\frac{\int_{x_{1}}^{x_{2}} \omega d \sigma}{V}, \text { and } \frac{d \eta}{d t}=\frac{\int_{x_{1}}^{x_{2}} \frac{d h}{d t} d \sigma}{2 V},
$$

which are expressions for the velocity of the center of gravity of the 
volume. The two integrals $\int_{x_{1}}^{x_{3}} \omega d \sigma$ and $\int_{x_{1}}^{x_{2}} \frac{d h}{d t} d \sigma$ are readily evaluated by means of eq 112 and 122 . Writing $h d x$ for $d \sigma$, we obtain

and

$$
\int_{x_{1}}^{x_{2}} \omega d \sigma=\sqrt{g H}\left[V+\frac{3}{4 H} \int_{x_{1}}^{x_{3}} h d \sigma+\frac{H^{2}}{6}\left(\frac{\partial h}{\partial x}\right)_{x_{1}}^{x_{2}}\right],
$$

$$
\int_{x_{1}}^{x_{2}} \frac{d h}{d t} d \sigma=-\sqrt{g H}\left[\frac{h^{3}}{4 H}+\frac{H^{2}}{6}\left\{h \frac{\partial^{2} h}{\partial x^{2}}-\left(\frac{\partial h}{\partial x}\right)^{2}\right\}\right]_{x_{1}}^{x_{2}} .
$$

When these expressions are substituted in eq 123 and 124, we obtain for the velocity components of the center of gravity

and

$$
\frac{d \xi}{d t}=\sqrt{g \bar{H}}\left[1+\frac{3 \eta}{2 H}+\frac{H^{2}}{6 V}\left(\frac{\partial h}{\partial x}\right)_{x_{1}}^{x_{2}}\right]
$$

$$
\frac{d \eta}{d t}=-\frac{\sqrt{g H}}{2 V}\left[\frac{h^{3}}{4 H}+\frac{H^{2}}{6}\left\{h \frac{\partial^{2} h}{\partial x^{2}}-\left(\frac{\partial h}{\partial x}\right)^{2}\right\}\right]_{x_{1}}^{x_{x}} .
$$

Equation 125 simplifies if the slopes $\partial h / \partial x$ vanish at the end points of the intumescence considered, or if the volume $V$ is large, yielding

$$
\left(\frac{d \xi}{d t}\right)^{2}=g(H+3 \eta) \text {. }
$$

Equation 126 likewise simplifies if applied to the entire body of an intumescence. If the intumescence is unlimited, $V$ is infinitely large, and the right-hand member vanishes. It also vanishes if the intumescence is limited, for then both $h$ and $\partial h / \partial x$ vanish at the anterior and posterior ends of the intumescence. Therefore, the center of gravity of an intumescence is maintained at a constant height above the primitive free surface, and the velocity of propagation of this center of gravity is constant. [5, p. 84].

\section{VARIATION IN ENERGY OF AN INTUMESCENCE}

It may be inferred from the invariability of $\eta$ that the integral $\int h d \sigma$, when applied to an entire intumescence, is independent of time; that is, the integral

$$
\int_{x_{0}}^{\infty} h^{2} d x
$$

is independent of time. When this integral is multiplied by $\rho g / 2$, the product represents the potential energy of the intumescence, eq 62 . On the other hand, the kinetic energy of the entire liquid is given by the double integral

$$
\rho / 2 \int_{x_{0}}^{\infty} d x \int_{0}^{H+h}\left(u^{2}+w^{2}\right) d z .
$$

The velocity components, $u$ and $w$, are given by eq 114 and 115 . From these equations, neglecting quantities of small order, we obtain

$$
\int_{0}^{H+h}\left(u^{2}+w^{2}\right) d z=g\left[h^{2}+\frac{h^{3}}{2 H}+\frac{H^{2}}{3} \frac{\partial}{\partial x}\left(h \frac{\partial h}{\partial x}\right)\right]
$$


which we multiply by $\rho d x / 2$ and integrate between the limits $x=x_{0}$ and $x=\infty$. The integral $h \partial h / \partial x$ vanishes at infinity and also, when the intumescence is limited, at $x_{0}$. It also vanishes at $x_{0}$ when the intumescence is unlimited, because this integral is negligible in comparison with the first two terms owing to the length of the intumescence. Thus the kinetic energy of the wave system is

$$
\frac{\rho g}{2} \int_{x_{0}}^{\infty} h^{2} d x+\frac{\rho g}{4 H} \int_{x_{0}}^{\infty} h^{3} d x .
$$

The total energy of wave, $E$, consequently, is

$$
\rho g E=\rho g \int_{x_{0}}^{\infty} h^{2} d x+\frac{\rho g}{4 H} \int_{x_{0}}^{\infty} h^{3} d x .
$$

It consists of two terms, of which the first is independent of time, and the second which is small in comparison with the other, may be shown to vary very slowly with time.

\section{THE MOMENT OF INSTABILITY OF AN INTUMESCENCE}

It was seen that every intumescence is characterized by two integrals, $E=\int h d \sigma$ and $V=\int d \sigma$, which are independent of time. (See eq 65, 69, and 88). In addition to these, there also exists another integral

$$
M_{s}=\int_{x_{0}}^{\infty}\left[\left(\frac{\partial h}{\partial x}\right)^{2}-\frac{3 h^{3}}{H^{3}}\right] d x,
$$

which is independent of time. The invariant character of this integral was discovered by Boussinesq, who referred to it as the moment of instability [5, p. 97].

We consider the integral

$$
M_{8}-\frac{6}{H^{2}} E=\int_{x_{0}}^{\infty}\left[\left(\frac{\partial h}{\partial x}\right)^{2}-\frac{3 h^{3}}{H^{3}}-\frac{6 h^{2}}{H^{2}}\right] d x,
$$

where $E$ is the integral representing the energy of the intumescence. Differentiating both members of the equation, we obtain, since $d E / d t=0$,

$$
\begin{aligned}
\frac{d M_{s}}{d t} & =-\left[\left\{\left(\frac{\partial h}{\partial x}\right)^{2}-\frac{3 h^{3}}{H^{3}}-\frac{6 h^{2}}{H^{2}}\right\} \omega+2 \frac{\partial h}{\partial x} \frac{\partial h}{\partial t}\right]_{x_{0}} \\
& -\int_{x_{0}}^{\infty} \frac{\partial h}{\partial t}\left(12 \frac{h}{H^{2}}+\frac{9 h^{2}}{H^{3}}+2 \frac{\partial^{2} h}{\partial x^{2}}\right) d x .
\end{aligned}
$$

The term in the brackets vanishes when the intumescence is limited; in the contrary case it is negligible in comparison with the integral, owing to the great length of the intumescence. Therefore,

$$
\frac{d M_{s}}{d t}=-\int_{x_{0}}^{\infty} \frac{\partial h}{\partial t}\left(12 \frac{h}{H^{2}}+\frac{9 h^{2}}{H^{3}}+2 \frac{\partial^{2} h}{\partial x^{2}}\right) d x,
$$

or, in view of eq 112 ,

$$
\frac{d M_{s}}{d t}=-\frac{12}{\sqrt{g H^{5}}} \int_{x_{0}}^{\infty} \frac{\partial h}{\partial t} h \omega d x
$$


Now, introducing $\partial h / \partial t$ from eq 90 , and then integrating, we obtain

$$
\frac{d M_{s}}{d t}=\frac{6}{\sqrt{g H^{5}}}(h \omega)_{x_{0}} .
$$

If the intumescence is limited, the right-hand member vanishes: if it is unlimited, this member is a quantity of the order which is neglected in this analysis. Therefore, to this approximation, the moment of instability of an intumescence is independent of time.

\section{SOLITARY WAVES}

The effect of height and curvature on the deformation of waves was discussed in the previous section on the basis of the assumption that deformation actually occurs. It may well be that configurations exist with appreciable height and curvature which are propagated with no deformation whatever. As a matter of fact, such waves were first discovered by experiment. The solitary wave of J. Scott Russell, consisting of a single elevation whose height may be of the same order of magnitude as the depth, may travel considerable distances without appreciable deformation $[6$, p. $505 ; 7$, p. $324 ; 9$, p. 289]. This property is called longevity and the law governing it may be derived from eq 106.

Putting new variables

$$
\begin{gathered}
(x-\omega t)=\zeta H, \\
h=\eta H,
\end{gathered}
$$

and

$$
\left(\frac{\omega^{2}}{g H}-1\right)=b
$$

into eq 106 , we get, since $\frac{\partial^{2}}{\partial t^{2}}=\omega^{2} \frac{\partial^{2}}{\partial x^{2}}$ on the basis of the assumption that the wave does not deform,

$$
\frac{\partial^{2}}{\partial \zeta^{2}}\left(6 b \eta-9 \eta^{2}-2 \frac{\partial^{2} \eta}{\partial \zeta^{2}}\right)=0
$$

which when integrated twice gives

$$
6 b \eta-9 \eta^{2}-2 \frac{\partial^{2} \eta}{\partial \zeta^{2}}+c_{1} \zeta+c_{2}=0 .
$$

Since $\eta$ and $\frac{\partial^{2} \eta}{\partial \zeta^{2}}$ are finite, $c_{1}=0$. If we restrict ourselves to the case where at $\zeta=\infty, \eta=0$ and $\frac{\partial^{2} \eta}{\partial \zeta^{2}}=0$, then $c_{2}=0$ and we have

$$
6 b \eta-9 \eta^{2}-2 \frac{d^{2} \eta}{d \xi^{2}}=0 .
$$

Multiplying by $\frac{d \eta}{d \zeta} d \zeta$ and integrating: 


$$
3 b \eta^{2}-3 \eta^{3}=\left(\frac{d \eta}{d \zeta}\right)^{2} .
$$

Denoting the maximum height of the wave by $h_{1}$, and the corresponding value of $\eta$ by $\eta_{1}$,

$$
\eta_{1}=\frac{h_{1}}{H}
$$

At the crest of the wave, $\eta=\eta_{1}$, and the wave surface is parallel to the bed, $d \eta / d \zeta=0$. Substituting these values in eq 138 we obtain the relation between $b$ and $\eta_{1}$,

$$
\eta_{1}=b=\left(\frac{\omega^{2}}{g H}-1\right) .
$$

Replacing this value of $b$ in eq 138:

$$
\left(\frac{d \eta}{d \zeta}\right)^{2}=3 \eta^{2} \eta_{1}-3 \eta^{3}
$$

and integrating twice:

$$
\eta=\eta_{1} \operatorname{sech}^{2}\left[\sqrt{\frac{3}{4} \eta_{1} \zeta}\right] .
$$

Reverting to the original variables, we obtain the equation of the wave profile

$$
h=h_{1} \operatorname{sech}^{2}\left[\sqrt{\frac{3 h_{1}}{4 H^{3}}}(x-\omega t)\right] .
$$

The free surface is symmetrical about the normal at $x=\omega t$, and approaches the primitive surface as $x-\omega t$ approaches $\pm \infty$ (fig. 2).

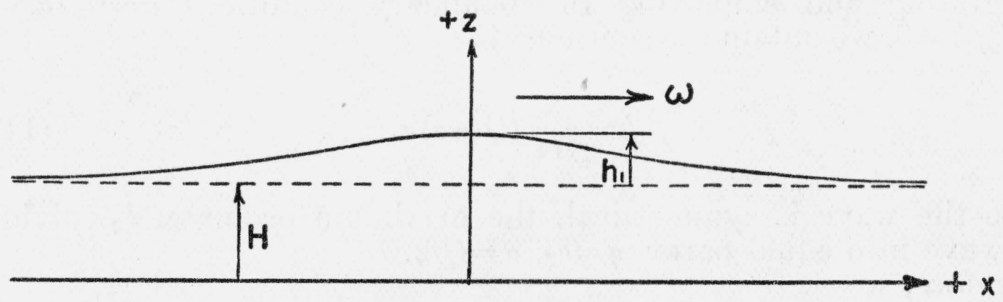

FIGURE 2.-Diagram of a solitary wave.

The velocity of propagation, $\omega$, which may be interpreted either as that of the volume element or height element since the wave does not deform, is obtained from eq 140:

$$
\omega^{2}=g\left(H+h_{1}\right),
$$

which is the formula adopted by Russell [7, p, 328; 8, p. 423], on empirical grounds and confirmed by the experiments of Bazin [6, p. $496,515]$. 
A comparison of theory and experiment, based upon 117 observations by Bazin [6, p. 510-514], shows the error of the formula to be $-0.91 \pm 5.10$ percent. Similar calculations based upon 77 observations by Russell $[8$, p. $440-442]$ give $+0.78 \pm 2.30$ percent. The \pm term indicates the standard deviation, and this usage will be followed in subsequent comparisons. It will be noted that in each case zero error is well within the limits determined by the standard deviation.

From eqs 127 and 143 we will now show that the center of gravity of a wave propagated without change of form is $h_{1} / 3$ above the primitive surface, where $h_{1}$ is the maximum height of the wave. Since the wave retains its form,

$$
\frac{d \xi}{d t}=\omega
$$

and is constant. Substituting from eq 143 in eq 127 , we obtain

$$
\eta=\frac{h_{1}}{3} \text {. }
$$

A relation connecting the height at any arbitrary point with the partial volumes of the wave on each side of the perpendicular at this point is derived as follows. By virtue of the relation $(1 / h)(\partial / \partial x)=\partial / \partial \sigma$, when $d h / d t$ is placed equal to zero, eq 122 yields the result:

$$
h^{3}\left(1+\frac{2 H^{3}}{3} \frac{d^{2} h}{d \sigma^{2}}\right)=\text { constant. }
$$

The constant is zero, since $h$ vanishes at infinity; therefore,

$$
1+\frac{2 H^{3}}{3} \frac{d^{2} h}{d \sigma^{2}}=0
$$

Integrating and employing the boundary conditions $\sigma=0, h=0$; $\sigma=Q, h=0$, we obtain the solution

$$
h=\frac{3}{4 H^{2}}(Q-\sigma) \sigma .
$$

Since the wave is symmetrical, the maximum ordinate, $h_{1}$, divides the wave into equal parts: $\eta=h_{2}, \sigma=Q / 2$.

Therefore,

$$
h_{1}=\frac{3 Q^{2}}{16 H^{3}},
$$

which is the relation between the maximum height of the wave and its total volume. It was demonstrated in section IV-2 that the potential and kinetic energies of an intumescence are equal, to within a small term of the order of $h^{3}$; consequently

$$
E=2 \eta Q=\frac{Q^{3}}{8 H^{3}} \text {. }
$$


From eq 148 and 149 we derive the following relations:

$$
h_{1}=\frac{3 \sqrt[3]{E^{2}}}{4 H}, \text { and } \frac{Q}{h_{1}}=\frac{8 H^{2}}{3 \sqrt[3]{E}}
$$

These relations will now be used to explain the phenomena of waves traveling up a channel of slowly decreasing depth. Considering eq 85 , we see that the quantity $c_{1}-c_{2}$ is small, since $d H / d x$ is small. Consequently, the reflected wave is negligible, and the energy of the transmitted wave remains sensibly constant. From eq 150 we deduce that $h_{1}$ continually increases, and $Q / h_{1}$ continually decreases. The wave gets higher and higher, and its base narrows continually. As a result, the wave becomes unstable and finally breaks. A quantitative analysis of this phenomenon will be given in section V-19.

\section{CNOIDAL WAVES}

In the theory developed thus far, extensive use has been made of the assumption that the liquid is undisturbed at infinity; that is, that the wave profile $x=f(h)$ vanishes at infinity together with all its derivatives. In the previous section this assumption was utilized in deriving eq 137, and it was found that the only wave fulfilling this condition and propagated without change of form was the solitary wave. We now wish to eliminate this restriction and determine the form of a wave of appreciable height, governed by eq 106 and propagated without change of form.

We therefore assume that at $h=0$, the derivatives $\partial h / \partial x, \partial^{2} h / \partial x^{2}$, ... do not all necessarily vanish. The constant $c_{2}$ is consequently retained in eq 136 , and we write

$$
6 b \eta-9 \eta^{2}-\frac{d^{2} \eta}{d \zeta^{2}}+3 c_{2}=0 .
$$

Multiplying both members of eq 151 by $(d \eta / d \zeta) d \zeta$ and integrating:

$$
\left(\frac{d \eta}{d \zeta}\right)^{2}=3 b \eta^{2}-3 \eta^{3}+3 c_{2} \eta+3 c_{1},
$$

where $3 c_{1}$ is another constant of integration. Since $d \eta / d \xi$ is real at $\eta=0$, then $c_{1}$ is positive, and if $\eta^{\prime}, \eta^{\prime \prime}, \eta^{\prime \prime \prime}$ are the roots of the polynomial, then

$$
\eta^{\prime} \eta^{\prime \prime} \eta^{\prime \prime \prime}=c_{1}
$$

Either all the roots are positive, or one is positive and two negative, or one is positive and two complex. There is at least one positive value of $\eta$, say $\eta^{\prime}$, for which $d \eta / d \zeta$ vanishes. If this is the only value of $\eta$ for which the profile is horizontal, then the wave profile must become infinite with $\zeta$, which is physically impossible. The third case is therefore excluded. If all the roots are positive, then the wave profile must be included between the extreme values, since otherwise it would become infinite. This requires that the wave surface be everywhere above the primitive surface, which is likewise physically impossible, and the first case is also excluded. Therefore $\eta^{\prime \prime}$ and $\eta^{\prime \prime \prime}$ are both negative. Writing the three roots $\eta_{1},-\eta_{2},-\eta_{3}, \eta_{3} \geqq \eta_{2}$, we have 


$$
\left(\frac{d \eta}{d \zeta}\right)^{2}=3\left(\eta_{1}-\eta\right)\left(\eta+\eta_{2}\right)\left(\eta+\eta_{3}\right)
$$

and

$$
\left.\begin{array}{l}
b=\eta_{1}-\eta_{2}-\eta_{3}, \\
c_{2}=-\eta_{2} \eta_{3}+\eta_{1} \eta_{3}+\eta_{1} \eta_{2}, \\
c_{1}=\eta_{1} \eta_{2} \eta_{3} .
\end{array}\right\}
$$

Introducing the new variable, $x$,

eq 153 becomes

$$
\eta=\eta_{1} \cos ^{2} \chi-\eta_{2} \sin ^{2} \chi
$$

where

$$
\beta\left(\frac{d \chi}{d \zeta}\right)=\sqrt{1-k^{2} \sin ^{2} \chi}
$$

$$
\beta=\sqrt{\frac{4}{3\left(\eta_{1}+\eta_{3}\right)}}=\sqrt{\frac{4 H}{3\left(h_{1}+h_{3}\right)}},
$$

and

$$
k^{2}=\frac{\eta_{1}+\eta_{2}}{\eta_{1}+\eta_{3}}=\frac{h_{1}+h_{2}}{h_{1}+h_{3}}
$$

Selecting $\zeta=0$ at the apex, then $\eta=\eta_{1}$ and $\chi=0$, and we have

$$
\zeta=\beta \int_{0}^{x} \frac{d \chi}{\sqrt{1-k^{2} \sin ^{2} \chi}}=\beta F(\chi, k)
$$

where $F$ is an incomplete elliptic integral of the first kind. Introducing Jacobian elliptic functions, since $\cos \chi=\operatorname{cn}(\xi / \beta, k)$, sin $\chi=$ $\operatorname{sn}(\zeta / \beta, k)$ and $\operatorname{sn}^{2} u+\operatorname{cn}^{2} u=1$, we have

$$
\eta=-\eta_{2}+\left(\eta_{1}+\eta_{2}\right) \mathrm{cn}^{2}\left(\frac{\zeta}{\beta}, k\right)
$$

or reverting to the original coordinates

$$
h=-h_{2}+\left(h_{1}+h_{2}\right) \mathrm{cn}^{2}\left[\sqrt{\frac{3\left(h_{1}+h_{3}\right)}{4 H^{3}}}(x-\omega t), \sqrt{\frac{h_{1}+h_{2}}{h_{1}+h_{3}}}\right] .
$$

This equation represents an infinite number of undulations of identical size and shape, each symmetrical about a vertical plane passing through the apex. The existence of these periodic waves moving without change of form was first indicated by Boussinesq $[10$, p. 392], and the name, cnoidal waves, from the cn function by analogy with sinusoidal, is due to Korteweg and de Vries [11].

The relative wave length, $\lambda / H$, is given by

$$
\frac{\lambda}{H}=2 \beta \int_{0}^{\pi / 2} \frac{d \chi}{\sqrt{1-k^{2} \sin ^{2} \chi}}=2 \beta F_{1}(k),
$$

$F_{1}$ being the complete elliptic integral of the first kind. 

have

The initial depth of the water in the channel being $H$, we must $\int_{0}^{\frac{\lambda}{H}} \eta d \xi=0$, or from eq 155 and eq 156

$$
2 \int_{0}^{\frac{\pi}{2}} \frac{\left(\eta_{1} \cos ^{2} \chi-\eta_{2} \sin ^{2} \chi\right) \beta}{\sqrt{1-k^{2} \sin ^{2} \chi}} d \chi=0,
$$

which from eq 158 reduces to

$$
\left(h_{1}+h_{3}\right) E_{1}(k)=h_{3} F_{1}(k),
$$

where $E_{1}$ is the complete elliptic integral of the second kind. The form of the waves is completely determined by $\lambda / H$, and $h_{1} / H$, the relative wave length and height of the crest above the initial surface of the water, since $h_{2} / H$ and $h_{3} / H$ are determined by eq 158,162 , and 163.

From eq 162 we see that the wave length of the undulations becomes infinite when $k=1$. From eq 163 if $k=1, h_{2}=h_{3}=0$. Since $\operatorname{cn}(\chi, 1)=\operatorname{sech} \chi$, then for $k=1$, eq 161 reduces to the equation of the solitary wave (eq 142), and the latter appears as a special case of the cnoidal wave.

From the definition of $b$ (eq 136) and from eq 154, we have

$$
\frac{\omega^{2}}{g H}=1+\frac{h_{1}}{H}-\frac{h_{2}}{H}-\frac{h_{3}}{H},
$$

which is the expression for the velocity of the wave profile. Since we are now dealing with an infinite number of undulations moving in a channel of finite depth, a question arises as to the meaning of the velocity of propagation. Stokes [12, p. 202], has given two definitions applicable to this case, and we adopt the second one; namely, the velocity of propagation is the velocity of the wave profile with respect to the center of inertia of the fluid mass included between two vertical planes separated by a distance equal to the wave length.

Writing this velocity as $\omega_{c}$ :

$$
\omega_{c}=\omega-\frac{1}{H \lambda} \int_{0}^{\lambda} U(H+h) d x,
$$

where $U$ is the mean velocity in a vertical section. If $U_{0}$ is the mean velocity in the section at $h=0$, we have from eq 95

$$
U(H+h)=h \omega+U_{0} H \text {. }
$$

Substituting in eq 165 , we have for the velocity of propagation

or

$$
\omega_{c}=\omega-U_{0}
$$

$$
\omega_{c}^{2}=\omega^{2}\left(1-2 \frac{U_{0}}{\omega}\right)
$$

We now evaluate $U_{0}$. Integrating eq 114 , we have

$$
U_{0}=u_{0}-\frac{H^{2}}{6} \frac{\partial^{2} u_{0}}{\partial x^{2}}, h=0
$$


$u_{0}$ being the velocity at the bottom. It has been assumed in the analysis that at $h=0$, the surface velocity $u_{1}$ vanishes (see the discussion of eq 41); hence from eq 114,

and

$$
u_{0}=\frac{H^{2}}{2} \frac{\partial^{2} u_{0}}{\partial x^{2}}, h=0,
$$

$$
U_{0}=\frac{H^{2}}{3}\left(\frac{\partial^{2} u_{0}}{\partial x^{2}}\right), h=0 .
$$

From eq 104, since we can write $\partial / \partial t=-\omega(\partial / \partial x)$, we obtain by differentiating twice with respect to $x$, and neglecting the term containing $\partial^{4} h / \partial x^{4}$ :

$$
\frac{\partial^{2} u_{0}}{\partial x^{2}}=\frac{g}{\omega}\left(\frac{\partial^{2} h}{\partial x^{2}}\right), h=0 .
$$

Substituting in eq 169 , we finally have

and eq 168 becomes

$$
U_{0}=\frac{1}{3} \frac{g H^{2}}{\omega} \frac{\partial^{2} h}{\partial x^{2}}, h=0
$$

$$
\omega_{c}^{2}=\omega^{2}\left[1-\frac{2 g H^{2}}{3 \omega^{2}}\left(\frac{\partial^{2} h}{\partial x^{2}}\right)_{h=0}\right],
$$

or from eq $151,154,164$, in terms of $h_{1}, h_{2}$, and $h_{3}$, within the approximation of the analysis, that is, neglecting $h_{1} h_{2} h_{3} / H^{3}$,

$$
\omega_{c}^{2}=g H\left[1+\frac{h_{1}}{H}\right]\left[1-\frac{h_{2}}{H}\right]\left[1-\frac{h_{3}}{H}\right] .
$$

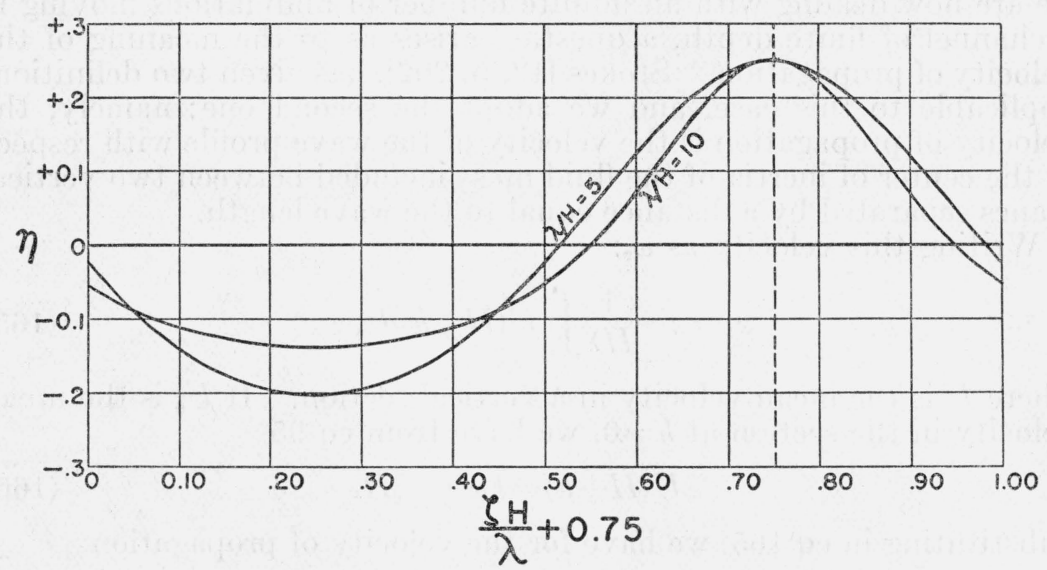

Figure 3.-Cnoidal waves.

Relative displacemen of the free surface as a function of the fractional part of the relative wave length.

This, then, is the velocity of propagation of the wave profile with respect to the center of inertia of the fluid mass included between two vertical planes separated by a distance equal to the wave length, $\lambda$.

For other treatments of this subject reference may be made to Boussinesq [10, p. 390], Korteweg and de Vries [11], and Lamb [4, p. 402]. 
Figures 3 and 4 represent two typical cnoidal waves. Figure 3 shows on an arbitrary scale one complete undulation for two values of $\lambda / H$, and figure 4 shows extended profiles of the same two waves at the correct height above the bottom, but compressed horizontally 5 times. In both cases, $h_{1} / H=0.25$.

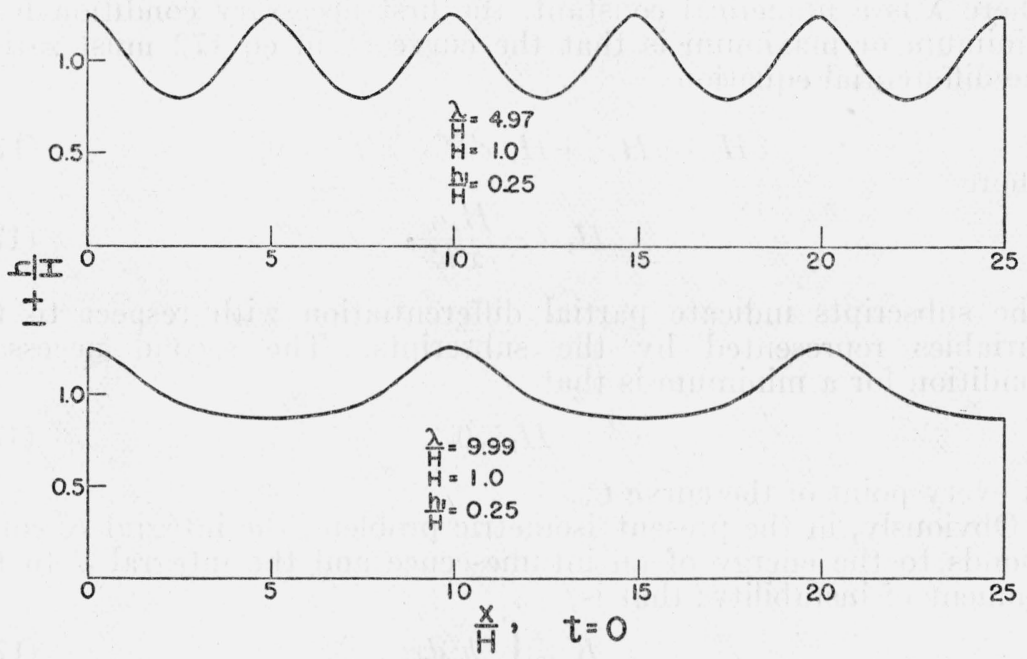

Figure 4.-Cnoidal waves.

Relative height above the bottom as a function of relative horizontal dimension.

10. MOMENT OF INSTABILITY OF A SOLITARY WAVE

Of all the intumescences of equal energy, the solitary wave is the only one for which the moment of instability

$$
M_{s}=\int_{x_{0}}^{\infty}\left[\left(\frac{\partial h}{\partial x}\right)^{2}-\frac{3 h^{3}}{H^{3}}\right] d x
$$

is a minimum.

The theorem may be proved by the methods of the calculus of variations for the solution of isometric problems. By an isometric problem, we mean one of the following kind:

Among all curves joining two points $\left(x_{0}, h_{0}\right)$ and $\left(x_{1}, h_{1}\right)$ for which the definite integral

$$
K=\int_{\mathrm{s}_{0}}^{\mathrm{s}_{1}} G\left(x, h, x^{\prime}, h^{\prime}\right) d s
$$

takes on a given value $l$, to determine the one

$$
\left.\begin{array}{rl}
C_{0}, x & =x(s), x_{0}=x\left(s_{0}\right) \\
h & =h(s), h_{0}=h\left(s_{0}\right)
\end{array}\right\}
$$

which minimizes (or maximizes) another definite integral

$$
J=\int_{\mathrm{s}_{0}}^{\mathrm{s}_{1}} F\left(x, h, x^{\prime}, h^{\prime}\right) d s .
$$


Here $s$ is a parametric variable, and the primes indicate differentiation with respect to $s$.

Putting

$$
H=F+\lambda G,
$$

where $\lambda$ is a numerical constant, the first necessary condition for a minimum or maximum is that the curve $C_{0}$ in eq 172 must satisfy the differential equation

where

$$
H_{x h^{\prime}}-H_{x^{\prime} h}+H_{1}\left(x^{\prime} h^{\prime \prime}-x^{\prime \prime} h^{\prime}\right)=0,
$$

$$
H_{1}=-\frac{H_{x^{\prime} h^{\prime}}}{x^{\prime} h^{\prime}}
$$

The subscripts indicate partial differentiation with respect to the variables represented by the subscripts. The second necessary condition for a minimum is that

$$
H_{1} \overline{>} 0
$$

at every point of the curve $C_{0}$.

Obviously, in the present isometric problem, the integral $K$ corresponds to the energy of an intumescence and the integral $J$ to the moment of instability; that is,

and

$$
K=\int_{x_{0}}^{\infty} h^{2} d x
$$

$$
J=\int_{x_{0}}^{\infty}\left[\left(\frac{\partial h}{\partial x}\right)^{2}-\frac{3 h^{3}}{H^{3}}\right] d x .
$$

We select the parametric variable $s$ so that

and

$$
\left.\begin{array}{l}
x=s, \\
h=h(s) .
\end{array}\right\}
$$

The above integrals, eq 178 and 179 , become

and

$$
K=\int_{s_{0}}^{\infty} h^{2} x^{\prime} d s,
$$

$$
J=\int_{s_{0}}^{\infty}\left(\frac{h^{\prime 2}}{x^{\prime}}-\alpha h^{3} x^{\prime}\right) d s,
$$

where $\alpha=3 / H^{3}$. Hence,

$$
\begin{aligned}
G & =h^{2} x^{\prime}, \\
F & =\frac{h^{\prime 2}}{x}-\alpha h^{3} x^{\prime}, \\
H_{1} & =\frac{2}{x^{\prime 3}},
\end{aligned}
$$

and

$$
H=\frac{h_{1}^{\prime 2}}{x^{\prime}}-\alpha h^{3} x^{\prime}+\lambda h^{2} x^{\prime} .
$$


The first necessary condition for a minimum, eq 175, after some simplifications, yields

$$
3 \alpha h^{2}-2 \lambda h+2 \frac{\partial^{2} h}{\partial x^{2}}=0,
$$

which also may be written as

$$
\frac{3}{4} h+\frac{1}{6} \frac{H^{3}}{h} \frac{\partial^{2} h}{\partial x^{2}}=\frac{1}{6} \lambda H^{3},
$$

if $\alpha$ is replaced by $3 / H^{3}$. Since the left-hand member is a constant, we see on comparing eq 188 with eq 112 that the intumescence having a maximum or a minimum moment of instability for a given energy is one that is propagated without change of form. The only wave which is propagated with constant velocity is the solitary wave. The second necessary condition, eq 177 , is likewise satisfied; hence the moment of instability is a minimum.

\section{MOMENT OF INSTABILITY AND WAVE FORMATION}

The frequent occurrence of the solitary wave, owing to its production by a broad class of different initial disturbances, was observed by Scott Russell [7, p. 351; 8, p. 423]. This frequent formation of the solitary wave has been explained by Boussinesq [5, p. 99; 10, p. 401], utilizing the theorem just proved-that the solitary wave is the only intumescence for which the moment of instability is a minimum. An integration would show that this minimum is

$$
\left(M_{s}\right)_{1}=\frac{27 E^{5 / 3}}{20 H^{4}},
$$

where $E$ is the energy of the wave and $H$ is the depth of the channel.

If one considers an intumescence of arbitrary form, and a solitary wave, both having the same energy, $E$, the excess of the integral $M_{s}$ of the intumescence over that of the solitary wave, $M_{s}-\left(M_{s}\right)_{1}$ may be regarded as a measure of the rapidity with which the intumescence will deform during its propagation and also of the extent of the deformation taking place. This is why Boussinesq named the integral the moment of instability.

If $M_{s}-\left(M_{s}\right)_{1}$ is small, the profile of the intumescence will oscillate about that of a solitary wave having the same energy, $E$. The form of the intumescence cannot change very markedly, as this would require $M_{s}$ to increase, contrary to the theory. Friction, which has been ignored in the theory, will damp out these oscillations and the intumescence will eventually assume the shape of a solitary wave.

It should be noted that there does not exist a solitary wave having an arbitrary energy, since there is a maximum height, above which a solitary wave cannot be propagated without breaking (see section $\mathrm{V}-19)$. This limiting factor serves to determine the class of disturbances which can produce a solitary wave. 


\section{NEGATIVE WAVES}

Let us now examine the circumstances governing the motion of a negative wave limited in extent and propagated in the positive $x$-direction, negative signifying that the surface is depressed. The longitudinal profile of such a wave in its simplest form will consist at the moment of the formation of the wave of a main concave portion in the center and two convex arcs at the posterior and the anterior ends of the wave. As long as the form is not altered, the center of gravity of the wave is located at one-third of its maximum depth; that is, $\eta=h_{1} / 3$. According to eq 127 , the velocity of propagation of this wave would be

$$
\omega^{2}=g\left(H-h_{1}\right),
$$

a law that is confirmed very nearly in the experiments of Bazin. Calculations based upon 18 observations, [6, p. 521] give as the error of the formula, $-1.54 \pm 2.09$ percent, where the \pm term indicates the standard deviation.

The initial simple form of the wave, however, is soon modified. In fact, eq 112 shows that since $h$ is negative, the third term is nega-
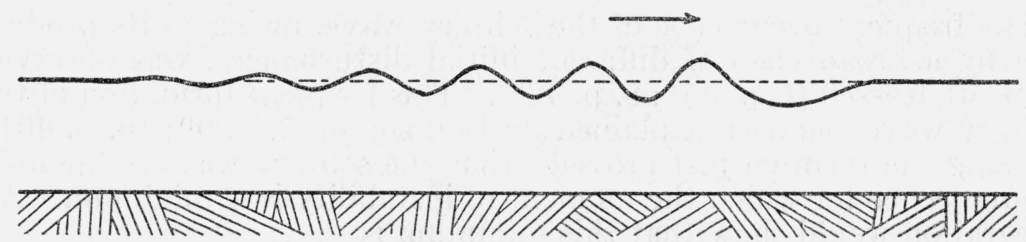

FIgURE 5.-Negative wave as observed by Bazin.

tive in the concave portion and positive in the convex portion. The second term is more negative in the concave portion than in the convex portion, since $h$ is smaller in absolute magnitude in the latter case. Thus the head of the depression, being both at a higher elevation than the center of the cavity and convex, while the latter is concave, is propagated faster than the center and becomes elongated. The tail of the wave also travels faster than the center for the same reasons and is therefore shortened as long as the requisite initial conditions obtain. However, the process of shortening cannot be indefinite. Furthermore, a convex portion is necessary for the continuity of the surface slope; hence the convex portion is finally raised above the level of the primitive free surface. Since $h$ is now positive, the last term of eq 112 is negative; and if either $h$ is small enough or $\partial^{2} h / \partial x^{2}$ large enough, it can exceed the second term, so that a condition is reached such that $\omega$ of the elevated convex portion is the same as $\omega$ of the lowest point of the depressed body. But the elevation thus produced at the posterior end of the main negative body of the wave requires an intermediate concave portion in order to join the free primitive surface with a continuous slope. Such a concave portion cannot exist and cannot be formed if its velocity of propagation is greater than that of the positive wave preceding it. Equation 112 indicates that if these velocities are to be equal, the convex positive wave must be followed by a negative wave. Continuing the argument, we see that a given negative wave is followed by a series of smaller waves, alternatively positive and negative, and entirely convex and 
concave, with inflection points on the level of the primitive surface (fig. 5). For additional figures, see [6, plate 2 , fig. $3 ; 7$, plate 52 , fig. 10].

\section{INTUMESCENCES OF FINITE HEIGHT BUT NEGLIGIBLE CURVATURE}

For an intumescence of finite height but having a free surface of negligible curvature, the expression of the velocity of propagation of volume element, eq 112, assumes the simple form

$$
\omega=\sqrt{g H}\left(1+\frac{3}{4} \frac{h}{H}\right)
$$

As an immediate consequence of this simplification, differential equation 90 can be integrated and the equation of the intumescence profile obtained. Introducing eq 191, eq 90 becomes

$$
\frac{\partial h}{\partial t}+\sqrt{g H}\left(1+\frac{3}{2} \frac{h}{H}\right) \frac{\partial h}{\partial x}=0,
$$

which possesses the integral

$$
x-\sqrt{g H}\left(1+\frac{3}{2} \frac{h}{H}\right) t=f(h),
$$

where $f(h)$ is an arbitrary function of $h$. The integral may be interpreted as follows: If $h$ is the elevation of the free surface at $x$ at time $t$, then the same elevation is reproduced at $x+\Delta x$ at time $t+\Delta t$; but, from eq 193:

$$
\Delta x=\sqrt{g H}\left(1+\frac{3}{2} \frac{h}{H}\right) \Delta t,
$$

and, therefore, the intumescence height is propagated with the velocity

$$
\omega_{1}=\sqrt{g \bar{H}}\left(1+\frac{3}{2} \frac{h}{H}\right)
$$

which is Airy's expression [4, p. 243] for the velocity of propagation for waves of finite height. It should be noted that eq 194 represents the velocity of propagation of the height of an element.

The arbitrary function, $f$, in eq 193 can be determined in two ways, depending on whether the canal is infinite in one or both directions. In the latter case, $h$ is usually given for all values of $x$ at some instant, $t$. If the relationship is in the form $h=f_{1}(x)$, we may invert the function and obtain $f_{2}(h)=x$, which is the desired function.

In the former case, assume the channel in communication with an infinitely large basin and that the water level of the basin is a known function of time, say, $h=F(t)$. Inverting this function, we have $t=F_{1}(h)$, and, if we take the point where the channel meets the basin as the origin of $x$, the arbitrary function is

$$
f(h)=-\sqrt{g H}\left(1+\frac{3}{2} \frac{h}{H}\right) F_{1}(h) .
$$


In intumescences of this type, the mean velocity, $U$, of the fluid particles in a cross section is

$$
U=\sqrt{g \bar{H}} \cdot \frac{h}{\bar{H}}\left(1-\frac{h}{4 H}\right),
$$

which follows from eq 96 and 191.

\section{HORIZONTAL CHANNEL IN COMMUNICATION WITH THE OCEAN}

Boussinesq has applied the method of the last article to a horizontal channel in communication with the ocean to determine the mean elevation of the ocean surface above the bed of the channel.

We may write for the elevation of the surface water of the ocean above the bed of channel,

$$
H+h=a\left(1+\alpha \sin \frac{2 \pi t}{T}\right), x=0,
$$

where $H$ is the depth of water in the channel at points so remote from the mouth that the disturbances of the ocean are not felt, $a$ is the elevation of the mean level of the surface waters of the ocean above the channel bed, $\alpha$ the ratio of the half amplitude of the tidal oscillations to the elevation, $a$, and $T$ the period of the tides. Using eq 171 , we obtain for the arbitrary function, $f(h)$, in eq 193,

$$
-f(h)=\sqrt{g \bar{H}}\left(1+\frac{3}{2} \frac{h}{H}\right) \frac{T}{2 \pi} \sin ^{-1} \frac{H+h-a}{a \alpha},
$$

and for the free surface of water in the channel,

$$
x=\sqrt{g H}\left(1+\frac{3}{2} \frac{h}{H}\right)\left(t-\frac{T}{2 \pi} \sin ^{-1} \frac{H+h-a}{a \alpha}\right) .
$$

After a certain length of time, the transients will die out and the total volume of water entering the channel during each period $T$ will be zero; that is,

$$
\int_{0}^{T} U(H+h) d t=0, x=0 .
$$

This condition permits the determination of $H$. Since $U(H+h)=\omega h$, eq 199 gives, substituting for $\omega$ from eq 191,

$$
\int_{0}^{T}\left(h+\frac{3}{4} \frac{h^{2}}{H}\right) d t=0 .
$$

Introducing $h$ from eq 196 and integrating, we obtain

$$
H-a-\frac{3}{4} \frac{(H-a)^{2}}{H}=\frac{3}{8} \frac{\alpha^{2} a^{2}}{H},
$$

which can be simplified further since $H-a$ is a small quantity:

$$
H-a=\frac{3}{8} \alpha^{2} a .
$$


This is the desired relation. The mean level of the surface water of the ocean is below the level of the water in the horizontal channel at large distances from the mouth by the small amount (3/8) $\alpha^{2} a$.

\section{WAVES DUE TO SUDDEN INCREASE OF DISCHARGE}

Let us examine the case in which a positive intumescence of infinite length is produced in a body of still water in a channel when a discharge of constant value is established at the entrance. After the initial disturbances have died down, the wave is of constant elevation, $h$, except in the anterior portion, and the front of the wave moves with a constant velocity, $\omega$. As the curvature of the layer representing the intumescence is negligible at points sufficiently removed from the front, the velocity of propagation of the wave front must be from eq 191,

$$
\omega=\sqrt{g\left(H+\frac{3}{2} h\right)},
$$

since $h / H$ is small.

Bazin's experiments verify this formula closely. From his data [6, p. 539-541], velocities were computed and were compared with the theoretical values. The mean values of $h$ and $H$ at the two points of the channel between which the wave traveled when its elapsed time was observed were substituted in the formula. The method of obtaining $h$ from the data is described on page 96 .

The data gave sufficient information to make 37 comparisons. Observations on eight intervals were rejected, since the observers reported that the wave had completely broken and reported $h$ as zero.

The average error of the theoretical values was $-0.98 \pm 2.71$ percent, where the \pm term indicates the standard deviation. These values were plotted as a function of $h / H$ in figure 6 , in an attempt to discover a systematic variation. The lines connect observations on the same wave in different intervals. The results were essentially negative, although there is a suggestion of an error changing in sign from minus to plus, as $h / H$ increases. It will be noted that the formula applies equally well to unstable waves on the point of breaking as well as to those having a definite $h_{1} / h$ ratio of $3 / 2$. (See fig. 9.)

St. Venant, using the principle of momentum, has derived for the celerity of this type of wave,

$$
\omega^{2}=g H\left(1+\frac{3}{2} \cdot \frac{h}{H}+\frac{1}{2} \frac{h^{2}}{H^{2}}\right),
$$

an expression which reduces to eq 202 when the last term is neglected.

\section{EFFECT OF CHANNEL VELOCITY DISTRIBUTION}

We may now consider a discharge wave superposed on a current and determine the effect of the velocity distribution in the current on the celerity of the wave.

We shall assume (1) that the profile of wave does not deform during the motion and (2) that at the two sections, each at a distance $s$ from the wave front, the velocity distributions are characteristic of the channel. (See fig. 7.) Let the mean velocities at these sections 


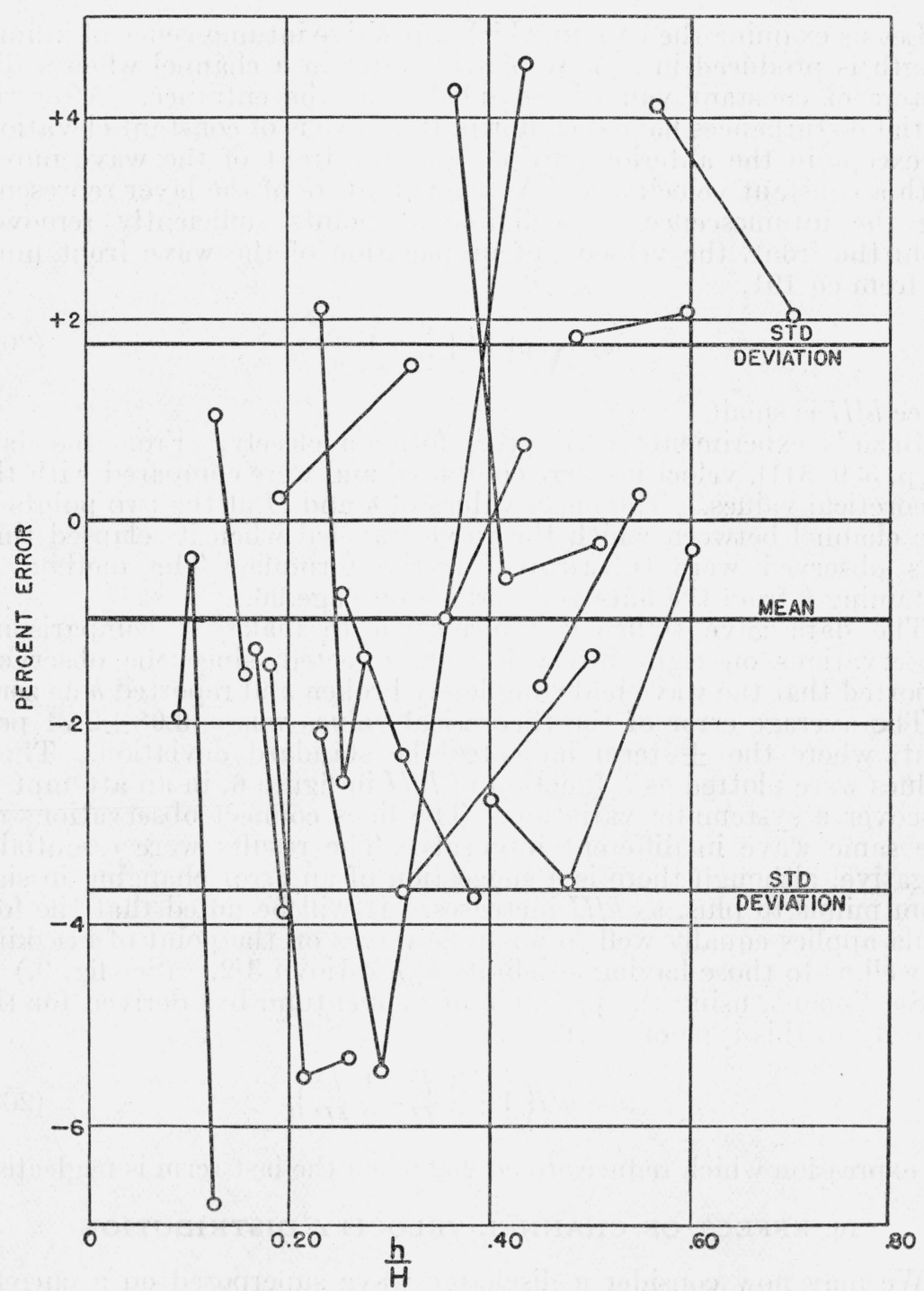

FIgURE 6.-Percentage of error of eq 202 compared with experiment, as a function of the relative height. 
be $U_{1}$ and $U_{2}$, the local velocities at any height $z$ be $u_{1}$ and $u_{2}$, and the depths $H$ and $H+h$. We consider the body of water included at the instant $t$ between the sections $A B$ and $D C$. The distance between $A B$ and the section containing the wave front is $s$; the distance between $D C$ and the wave front is $s+\omega \Delta t$, where $\omega$ is the celerity of the wave, and $\Delta t$ an interval of time. The momentum of the body of

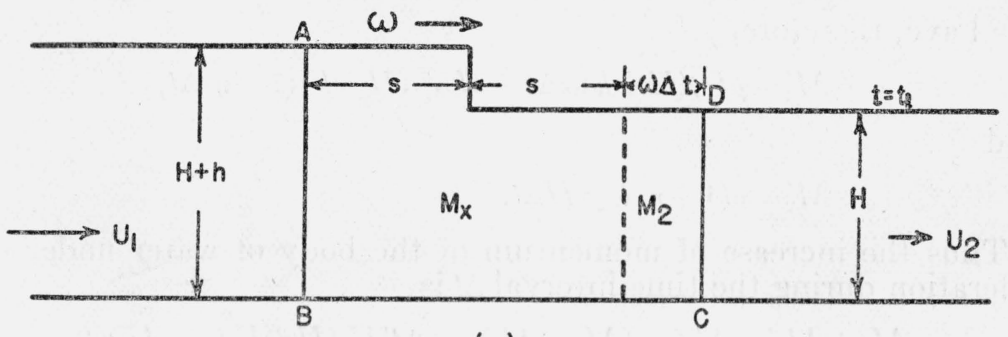

(a)

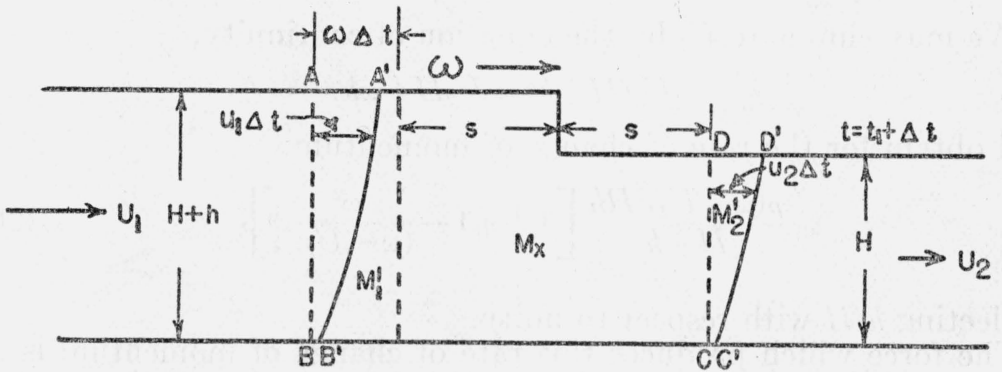

(b)

Figure 7.-Diagram illustrating the effect of channel velocity distribution.

(a) Configuration at time $t_{1} ;(b)$ Configuration at time $t_{1}+\Delta t$.

water thus delimited may be denoted by $M_{x}+M_{2}$, where $M_{2}$ is given by

$$
M_{2}=\rho \omega \Delta t \int_{0}^{H} u_{2} d z=\rho \omega \Delta t U_{2} H .
$$

At the instant of time $t+\Delta t$, the particles originally at $A B$ have reached the position $A^{\prime} B^{\prime}$, and the particles originally at $D C$ have reached the position $D^{\prime} C^{\prime}$. (See fig. 7.) The liquid contained between $A^{\prime} B^{\prime}$ and $D^{\prime} C^{\prime}$ has the momentum $M_{x}+M^{\prime}{ }_{1}+M^{\prime}{ }_{2}$, where

$$
M_{1}^{\prime}=\rho \int_{0}^{H+h}\left(\omega \Delta t-u_{1} \Delta t\right) u_{1} d z
$$

and

$$
M_{2}^{\prime}=\rho \int_{0}^{H} u_{2}^{2} \Delta t d z .
$$

Introducing the Boussinesq number, $\eta$, the coefficient of the excess of the mean of the velocities squared over the square of the mean velocity, which we assume to be unaffected by depth, 


$$
\int_{0}^{H+h} u_{1}^{2} d z=(1+\eta) U_{1}^{2}(H+h),
$$

and

$$
\int_{0}^{H} u_{2}^{2} d z=(1+\eta) U_{2}^{2} H
$$

We have, therefore,

$$
M_{1}^{\prime}=\rho U_{1}(H+h) \omega \Delta t-\rho U_{1}^{2}(H+h)(1+\eta) \Delta t,
$$

and

$$
M_{2}^{\prime}=\rho(1+\eta) U_{2}^{2} H \Delta t .
$$

Thus the increase of momentum of the body of water under consideration during the time interval $\Delta t$ is

$$
\begin{gathered}
M_{x}+M_{1}^{\prime}+M_{2}^{\prime}-\left(M_{x}+M_{2}\right)=\rho \Delta t\left[U_{1}(H+h)\left(\omega-U_{1}\right)+\right. \\
\left.U_{2} H\left(U_{2}-\omega\right)-\eta\left\{U_{1}^{2}(H+h)-U_{2}^{2} H\right\}\right] .
\end{gathered}
$$

We may eliminate $U_{1}$ by the equation of continuity,

$$
U_{1}(H+h)=U_{2} H+\omega h,
$$

and obtain for the rate of change of momentum:

$$
\frac{\rho\left(\omega-U_{2}\right)^{2} H h}{H+h}\left[1+\eta\left\{1-\frac{\omega^{2}}{\left(\omega-U_{2}\right)^{2}}\right\}\right]
$$

neglecting $h / H$ with respect to unity.

The force which produces this rate of change of momentum is the difference between the total hydrostatic pressures acting on the sections $A B$ and $D C$, which is

$$
\rho g H h\left[1+\frac{h}{2 H}\right] .
$$

Equating 206 and 207, and assuming that the effect of the $\eta$ term is small, we obtain

or

$$
\left(\omega-U_{2}\right)^{2}=g H\left(1+\frac{3}{2} \frac{h}{H}+\frac{h^{2}}{2 H^{2}}\right)\left[1-\eta\left\{1-\frac{\omega^{2}}{\left(\omega-U_{2}\right)^{2}}\right\}\right],
$$

$$
\omega=U_{2} \pm \sqrt{g H}\left(1+\frac{3}{4} \frac{h}{H}\right)\left(1-\frac{\eta}{2}\left[1-\frac{\omega^{2}}{\left(\omega-U_{2}\right)^{2}}\right]\right) .
$$

This is the expression for the celerity of a discharge wave or a depression wave, which takes into account the height, $h$, and $u_{2}$, the channel velocity distribution. For a wave moving downstream the plus sign holds; for a wave moving upstream the negative sign holds. For a positive wave $h$ represents an elevation and is positive; for a negative wave $h$ represents a depression and is negative.

Equation 208 can be further simplified when the approximation $\omega=U_{2} \pm \sqrt{g H}$ is used. Accordingly, we obtain for a wave moving downstream 


$$
\omega=U_{2}-\sqrt{g H}\left(1+\frac{3}{4} \frac{h}{H}\right)\left(1+\frac{\eta}{2}\left[\frac{U_{2}^{2}}{g H}+\frac{2 U_{2}}{\sqrt{g H}}\right]\right),
$$

and for a wave moving upstream

$$
\omega=U_{2}-\sqrt{g H}\left(1+\frac{3}{4} \frac{h}{H}\right)\left(1+\frac{\eta}{2}\left[\frac{U_{2}^{2}}{g H}-\frac{2 U_{2}}{\sqrt{g H}}\right]\right) .
$$

It is now seen that the effect of velocity distribution is appreciable only when the ratio $\mathrm{C}_{2}^{2} / g H$ is larger than unity, and for the ordinary case, in which $U_{2}^{2} / g H$ is a small fraction, this effect is negligible.

\section{INITIAL WAVES}

Bazin observed that in waves of constant discharge moving in still water, the initial part of the wave is made up of a series of relatively short, alternately convex and concave, intumescences. (See fig. 8.)
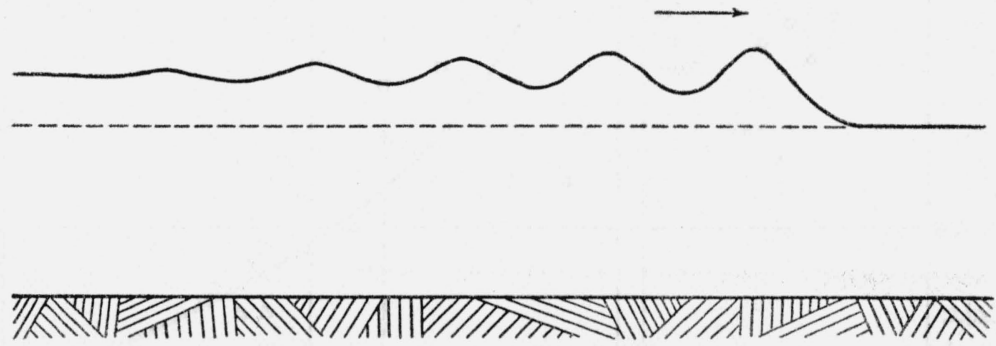

FIGURe 8.-Initial wave as observed by Bazin.

The foremost intumescence is convex, and its crest is at a higher lever than that of the constant elevation of the free surface of the main intumescence. Bazin refers to this initial part as the initial wave. The mechanical basis of the phenomenon was discussed by Boussinesq in the following manner, which is similar to the treatment in section $\mathrm{V}-12$.

At the head of the intumescence it is not possible for the depth to equal the depth of the main layer. The anterior part of the wave being convex, the curvature $\partial^{2} h / \partial x^{2}$ is negative and according to eq 112 ,

$$
\omega=\sqrt{g H}\left(1+\frac{3}{4} \frac{h}{H}+\frac{H^{2}}{6 h} \frac{\partial^{2} h}{\partial x^{2}}\right),
$$

the velocity of propagation there is less than that part of the layer which follows. This layer moves with a greater velocity and thus overruns the anterior portion of the wave until the augmented height compensates for the effect of the negative curvature. This explains the formation of the initial wave. It is known that the height of the initial wave tends to oscillate around a height equal to $3 / 2$ of the height, $h$, of the long layer. The height of the initial wave will increase until the velocity of propagation of its center of gravity becomes sensibly equal to that of the layer. But the form of this wave indicates that the center of gravity, $\eta$, is to be placed at $1 / 3$ of its height, $h_{a}$; and, on the other hand, eq 127, which is applicable in the present 198881-39-7 
case, gives for the square of the velocity of the propagation, $g\left(H+h_{a}\right)$. Equating this to the square of the velocity of the layer, $g(H+3 h / 2)$, eq 202 , it is seen that $h_{a}=3 h / 2$.

The ratio $h_{1} / h=3 / 2$ has been investigated by Bazin [6]. The experiments were made in a rectangular channel having a bottom slope of 1.5 in 1,000. The percentage change in depth being the least at the deep end, where the waves were generated, the motion at this end will nearly approximate flow in a channel of uniform depth. Calcu-

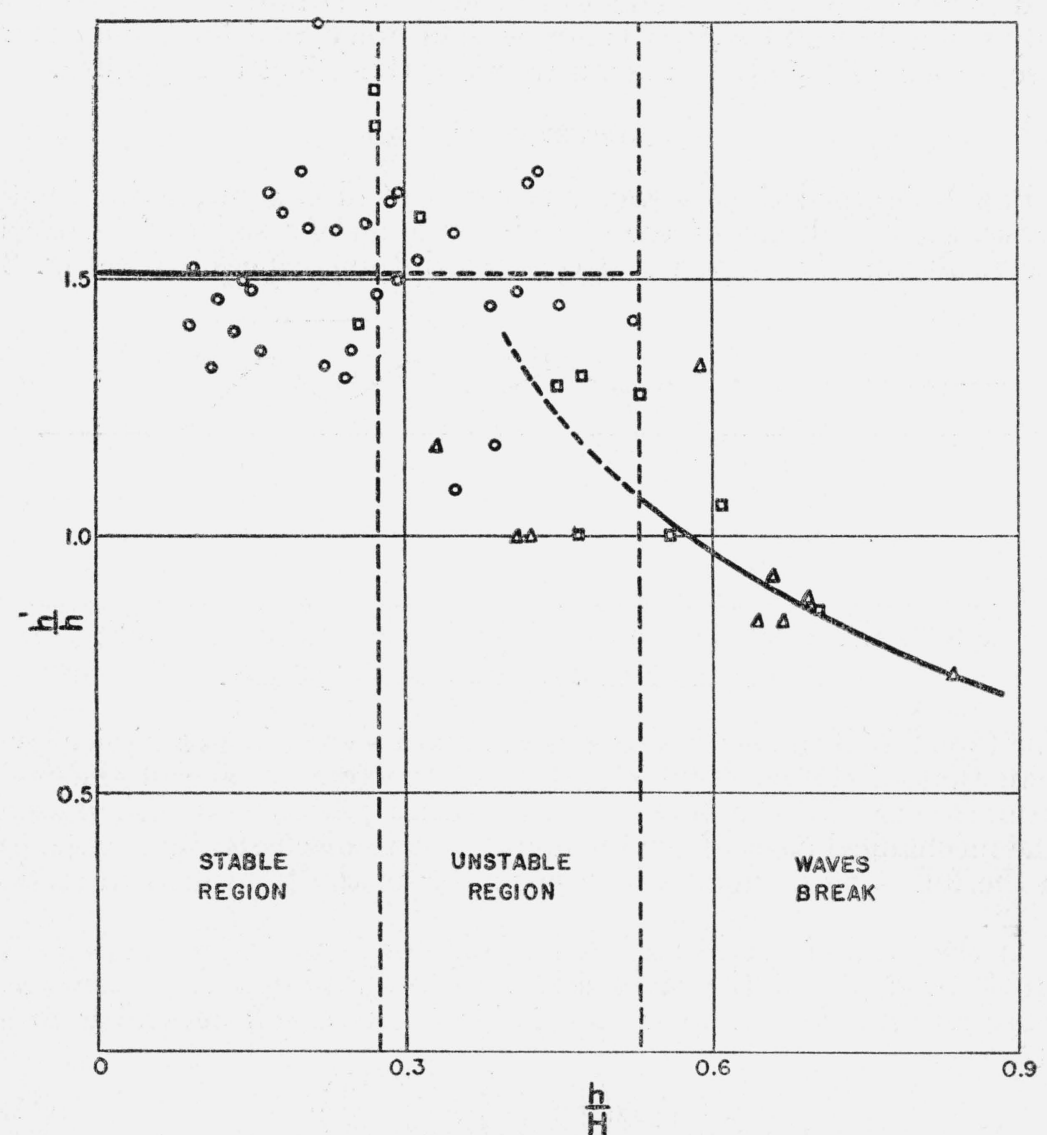

FIGURE 9.-Relative height of the head of an initial wave as a function of the relative depth of the main layer.

lations made by the authors from data taken at his end of the channel $\left[6\right.$, p. 542] give $h_{1} / h=1.61 \pm 0.18$. Bazin $[6$, p. 550] considers $3 / 2$ to be the approximate value.

Inasmuch as the waves are found to break as they ascend the channel if the original depth of the water is small enough, it is instructive to consider this aspect of the phenomenon. Observations were taken on the wave at four points in the channel. The complete data $\left[6\right.$, p. 539-541] have been employed to plot $h_{1} / h$ as a function of the dimensionless ratio $h / H$, the relative height of the wave layer. The average value of $h$ was utilized in order to approximate the 
steady height of the layer, eliminating the undulations behind the head of the wave. These data are represented in figure 9. The circles represent observations on waves which have no tendency to break; the squares, those in which breaking is imminent; the triangles, those in which breaking has occurred. An average of the 33 points with circles gives $h_{1} / h=1.51 \pm 0.17$. Although the data do not provide a clearly defined function, the following conclusions seem justified.

1. As would be expected, for increasing $h / H$ the waves become unstable and eventually break. The critical value is about 0.275 .

2. The value $h_{1} / h=1.5$ is a reasonable approximation in the stable region.

3 . The ratio $h_{1} / h$ definitely decreases with increasing $h / H$, outside the stable region.

Recent experiments $[2$, p. $158-159]$ indicate that $h_{1} / h$ is a function of $h / H$ in the stable region, increasing from 1 to 2 at the critical value 0.28 , then decreasing asymptotically toward unity in the unstable region.

Where the initial wave joins the main layer, the condition of continuity of slope requires a concave curve, that is, a curve for which $\partial^{2} h / \partial x^{2}$ is positive. The positive curvature would entail a velocity of propagation greater than that of the layer as a whole, unless $h$ is less than in the remainder of the layer. Hence a depression is produced immediately following the initial wave.

Continuing the argument, it is seen that the initial wave is followed by convexities and concavities all above the undisturbed surface. Because of the internal friction of the liquid, the heights of these convexities and concavities decrease in the posterior direction until they become insensible. The inflexion points where $\partial^{2} h / \partial x^{2}$ vanish are in a plane containing the free surface of the main layer.

\section{FAVRE'S EXPERIMENTS ON THE UNDULATIONS IN A POSITIVE SURGE}

In 1934, Favre [2] made an elaborate series of experiments on translation waves. Several of these experiments were undertaken to investigate the undulations composing the head of a discharge wave [2, ch. IV]. The experiments established the following facts:

1. The undulations are not formed immediately, but require a certain amount of time to establish themselves.

2. After the undulations are formed, they are of similar size and shape.

3. The final configuration is a stable one.

4. The height of the undulation at $x$ is independent of $\theta$, the time in which the flow is established, for a considerable range of values of $x$, provided $\theta \omega / x<0.2$. In other words, within certain limits, a discharge which is not created instantaneously will behave like a theoretically sudden increase of flow.

5 . The wave length of the undulations depends only on the mean height of the layer above the primitive surface. The former decreases as the latter increases.

These results suggest that the undulations composing the head of a discharge wave have a very general character, in the same sense that the solitary wave has a general character. Within certain limits, they are formed independently of the rate at which the discharge is 
increased, and depend only on the final discharge rate; they assume a stable configuration after a short initial interval, that is, they are propagated without change of form. In section V-9, it was mathematically demonstrated that the only wave propagated without change of form is the cnoidal wave, which includes the solitary wave as a special limiting case. It is also true that the solitary wave is a special case of the discharge wave from the physical point of view. The solitary wave may be formed by a sudden increase of discharge which soon decreases to zero, while the discharge wave is caused by a sudden increase of discharge which is maintained. All these considerations inevitably suggest the question: Are the undulations composing the head of a discharge wave cnoidal waves?

A comparison of figure 4 with Favre's figure 48 [2, p. 156] supports an affirmative answer. The wave profiles are very similar. However, there is a more significant comparison which may be made, namely, one between the theoretical velocity of propagation, $\omega_{c}$, and that observed by experiment. This is accomplished by using eq 167 and 170. From the values of $\eta_{1}$ and $\lambda / H$ given by the experimental data, we determine $\eta_{3}, k$, and $\eta_{2}$ by eq 163,162 , and 158 , and then calculate $\omega_{c}$ from eq 170. Equation 191 written in the form

$$
\omega=\sqrt{g H^{\prime}}\left(1+\frac{3}{4} \frac{h^{\prime}}{H^{\prime}}\right),
$$

is used to compute $\omega$. This equation, in the form of eq 202, has already been verified in section $\mathrm{V}-15$. In order to calculate $U_{0}$, we write

$$
\omega h^{\prime}=U_{0}\left(H^{\prime}+h^{\prime}\right),
$$

where $h^{\prime}$ is the average height of the layer, and $H^{\prime}$ is the undisturbed depth of the liquid. It should be noted that this $H^{\prime}$ is different from the $H$ in eq 170 . $U_{0}$ is computed from eq 211 with the assistance of eq 191. From eq 167 we have $\omega_{c}=\omega-U_{0}$. These two values are compared in the following tables for nine nonbreaking waves. Table 1 gives the experimental data from Favre [2, p. 157], and table 2 gives the results of the calculations. The explanation of the symbols is clearly indicated in figure 10.

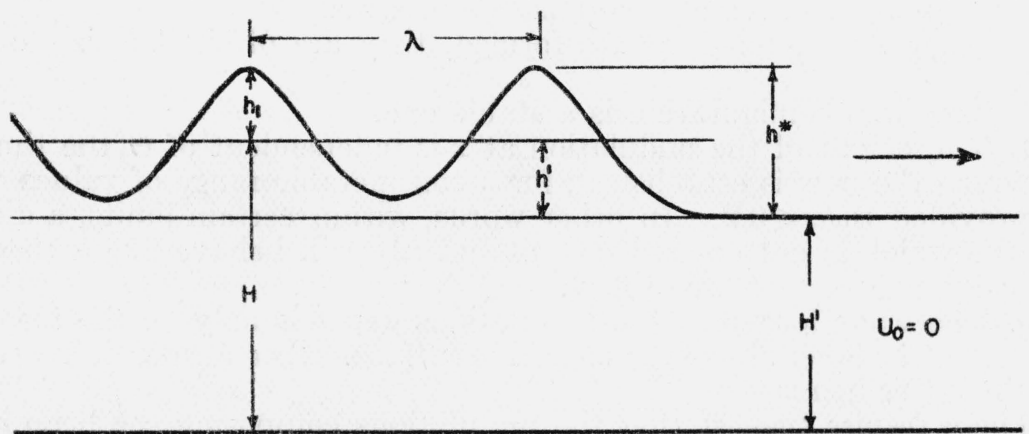

FIGURE 10.-Diagram illustrating the notation used in the studies of the positive surge. 
TABLE 1.-Favre's experimental data on positive surges

\begin{tabular}{|c|c|c|c|c|c|c|c|c|c|}
\hline \multirow{2}{*}{ Experiment No. } & \multirow{2}{*}{$\begin{array}{c}\text { Undis- } \\
\text { turbed } \\
\text { depth, } \\
H^{\prime}\end{array}$} & \multirow{2}{*}{$\begin{array}{c}\text { Mean } \\
\text { height } \\
\text { of layer } \\
h^{\prime}\end{array}$} & \multicolumn{4}{|c|}{ Crest height, $h *$, } & \multirow{2}{*}{$\underset{h^{*}}{\text { Mean }}$} & \multirow{2}{*}{$\begin{array}{l}\text { Wave } \\
\text { length }\end{array}$} & \multirow{2}{*}{$h^{\prime} / H^{\prime}$} \\
\hline & & & $\begin{array}{c}\text { 1st } \\
\text { wave }\end{array}$ & $\begin{array}{c}2 \mathrm{~d} \\
\text { wave }\end{array}$ & $\begin{array}{c}3 d \\
\text { wave }\end{array}$ & $\begin{array}{c}\text { 4th } \\
\text { wave }\end{array}$ & & & \\
\hline $8 \ldots$ & $\begin{array}{l}\text { cm } \\
20.55 \\
20.22 \\
20.19\end{array}$ & $\begin{array}{l}c m \\
2.29 \\
3.08 \\
4.08\end{array}$ & $\begin{array}{l}c m \\
3.90 \\
6.00 \\
8.10\end{array}$ & $\begin{array}{l}c m \\
3.60 \\
6.00 \\
8.00\end{array}$ & $\begin{array}{c}c m \\
-5.90\end{array}$ & \begin{tabular}{|c|}
$\mathrm{cm}$ \\
-1 \\
-2
\end{tabular} & $\begin{array}{l}\text { cm } \\
3.75 \\
5.97 \\
8.05\end{array}$ & $\begin{array}{l}c m \\
222 \\
194 \\
192\end{array}$ & $\begin{array}{r}0.111 \\
.152 \\
.202\end{array}$ \\
\hline 1210 & $\begin{array}{l}20.38 \\
20.57 \\
10.78\end{array}$ & $\begin{array}{l}4.84 \\
5.72 \\
0.86\end{array}$ & $\begin{array}{r}9.80 \\
11.70 \\
1.30\end{array}$ & $\begin{array}{r}8.70 \\
11.40 \\
1.20\end{array}$ & $\begin{array}{r}9.90 \\
10.90 \\
1.00\end{array}$ & 1.05 & $\begin{array}{r}9.47 \\
11.33 \\
1.14\end{array}$ & $\begin{array}{l}185 \\
176 \\
119\end{array}$ & $\begin{array}{l}.238 \\
.278 \\
.080\end{array}$ \\
\hline 22 (21 & $\begin{array}{l}10.73 \\
10.79 \\
10.74\end{array}$ & $\begin{array}{l}1.50 \\
2.48 \\
3.02\end{array}$ & $\begin{array}{l}2.80 \\
4.95 \\
6.25\end{array}$ & $\begin{array}{l}2.70 \\
4.70 \\
6.10\end{array}$ & $\begin{array}{l}2.85 \\
4.70 \\
5.70\end{array}$ & $\begin{array}{l}2.70 \\
4.45 \\
6.10\end{array}$ & $\begin{array}{l}2.76 \\
4.70 \\
6.04\end{array}$ & $\begin{array}{r}101 \\
91 \\
86\end{array}$ & $\begin{array}{r}.140 \\
.230 \\
.281\end{array}$ \\
\hline
\end{tabular}

TABLE 2.-Velocity comparisons between cnoidal waves and positive surges

\begin{tabular}{|c|c|c|c|c|c|c|c|c|c|c|c|c|}
\hline $\begin{array}{l}\text { Experi. } \\
\text { ment No. }\end{array}$ & $\begin{array}{c}h_{1}=\text { mean } \\
h^{*}-h^{\prime}\end{array}$ & $H=H^{\prime}+h^{\prime}$ & $\lambda / H$ & $\eta_{1}$ & $\eta / 2$ & $\eta_{8}$ & $\sin ^{-1} k$ & $\omega$ & $U_{0}$ & $\omega-U_{0}$ & $\omega_{0}$ & $\begin{array}{l}\text { Per- } \\
\text { cent- } \\
\text { age of } \\
\text { differ- } \\
\text { ence }\end{array}$ \\
\hline $\begin{array}{l}4 \\
6 \\
8\end{array}$ & $\begin{array}{c}c m \\
1.46 \\
2.89 \\
3.97\end{array}$ & $\begin{array}{l}c m \\
22.84 \\
23.30 \\
24.27\end{array}$ & $\begin{array}{l}9.72 \\
8.33 \\
7.91\end{array}$ & $\begin{array}{c}0.064 \\
.124 \\
.164\end{array}$ & $\begin{array}{r}0.052 \\
.094 \\
.118\end{array}$ & $\begin{array}{r}0.142 \\
.197 \\
.222\end{array}$ & $\begin{array}{l}48.6^{\circ} \\
55.4^{\circ} \\
58.8^{\circ}\end{array}$ & $\begin{array}{r}\mathrm{cm} / \mathrm{sec} \\
153.8 \\
156.9 \\
162.0\end{array}$ & $\begin{array}{c}c m / s e c \\
15.4 \\
20.7 \\
27.2\end{array}$ & $\begin{array}{c}c m / s e c \\
138.4 \\
136.2 \\
134.8\end{array}$ & $\begin{array}{c}\mathrm{cm} / \mathrm{sec} \\
139.2 \\
136.7 \\
137.9\end{array}$ & $\begin{array}{r}+0.58 \\
+.37 \\
+2.30\end{array}$ \\
\hline $\begin{array}{l}10 \\
12 \ldots \\
21\end{array}$ & $\begin{array}{l}4.63 \\
5.61 \\
0.28\end{array}$ & $\begin{array}{l}25.22 \\
26.29 \\
11.64\end{array}$ & $\begin{array}{r}7.34 \\
6.69 \\
10.22\end{array}$ & $\begin{array}{l}.184 \\
.213 \\
.024\end{array}$ & $\begin{array}{l}.126 \\
.157 \\
.022\end{array}$ & $\begin{array}{l}.257 \\
.307 \\
.126\end{array}$ & $\begin{array}{l}58.1^{\circ} \\
57.5^{\circ} \\
33.6^{\circ}\end{array}$ & $\begin{array}{l}166.7 \\
171.7 \\
109.0\end{array}$ & $\begin{array}{r}32.0 \\
37.4 \\
8.0\end{array}$ & \begin{tabular}{|l|}
134.7 \\
134.3 \\
101.0
\end{tabular} & $\begin{array}{l}137.9 \\
135.2 \\
100.0\end{array}$ & $\begin{array}{r}+2.38 \\
+0.67 \\
-.99\end{array}$ \\
\hline $\begin{array}{l}22 \\
23 \\
24\end{array}$ & $\begin{array}{l}1.26 \\
2.22 \\
3.02\end{array}$ & $\begin{array}{l}12.23 \\
13.27 \\
13.76\end{array}$ & $\begin{array}{l}8.26 \\
6.86 \\
6.25\end{array}$ & $\begin{array}{l}.103 \\
.167 \\
.219\end{array}$ & $\begin{array}{l}.081 \\
.129 \\
.166\end{array}$ & $\begin{array}{l}.198 \\
.289 \\
.349\end{array}$ & $\begin{array}{l}51.5^{\circ} \\
53.7^{\circ} \\
55.4^{\circ}\end{array}$ & $\begin{array}{l}113.4 \\
120.6 \\
124.3\end{array}$ & $\begin{array}{l}13.9 \\
22.5 \\
27.3\end{array}$ & $\begin{array}{l}99.5 \\
98.1 \\
97.0\end{array}$ & $\begin{array}{l}98.7 \\
97.0 \\
94.5\end{array}$ & $\begin{array}{r}-.80 \\
-1.12 \\
-2.58\end{array}$ \\
\hline
\end{tabular}

The average percentage error of $\omega_{c}$ as compared to $\omega-U_{0}$ is +0.09 \pm 1.53 , where the \pm term indicates the standard deviation. This is further evidence that the undulations in the head of a discharge wave are cnoidal waves. Inasmuch as these experiments were not intended to shed light on this particular problem, other points of verification cannot be easily attempted. An experiment made especially to investigate this problem would be more suitable.

\section{THEORY OF BREAKERS}

As was indicated at the end of section V-8, there is a limiting height for a solitary wave, dependent on the depth of the liquid in the channel. If an attempt is made to form a higher wave, it breaks at the crest. If $\omega$ is the velocity of propagation of the solitary wave, and $u_{1}$ is the absolute velocity of the liquid particle at the apex, that is, at the point $h=h_{1}$, it is evident that the limiting height is represented by the relation

$$
u_{1}-\omega=0 \text {, }
$$

since if $u_{1}>\omega$, the particle at the crest of the wave is traveling faster than the wave itself, and consequently the wave is breaking. 
Equating eq 112 and 143 and neglecting terms in $\left(h_{1} / H\right)^{2}$ or higher powers, we obtain for the curvature at the apex of the wave

$$
\left(\frac{\partial^{2} h}{\partial x^{2}}\right)_{h=h_{1}}=-\frac{3}{2} \frac{h_{1}^{2}}{H^{3}}
$$

Substituting this value in eq 116 , putting $z=H+h_{1}$ and neglecting terms of the order of $(h / H)^{4}$, the resulting velocity of the particle is

$$
u_{1}=\sqrt{g H}\left\{\frac{h}{H}+\frac{3}{2}\left(\frac{h}{H}\right)^{3}\right\} .
$$

Putting this value and that from eq 143 in eq 212, the limiting height is a root of the equation

$$
\frac{h_{1}}{H}+\frac{3}{2}\left(\frac{h_{1}}{H}\right)^{3}=\sqrt{1+\frac{h_{1}}{H}}
$$

The only positive real root of this equation is $h_{1} / H=0.731$, and this is the limiting height of the solitary wave.

This figure is confirmed reasonably well by experiment. Calculations made by the authors from Bazin's data [6, p. 510-514] give 0.71. Scott Russell [7, p. $352 ; 8$, p. $425,426,445,450]$ surmised that the wave broke when its height was approximately equal to the depth, and McCowan [13, p. 56] states that for his experiments 0.75 is a closer approximation for the relative elevation at the breaking point.

The above result may now be applied to the ultimate breaking of waves rolling in on a gently sloping bottom. Assuming that the waves are solitary, we obtain from eq 148 the following limiting condition involving the volume $Q$ :

or

$$
\frac{3 Q^{2}}{16 H^{4}}=\frac{h_{1}}{H}=0.73
$$

$$
H=0.71 \sqrt{Q} .
$$

This gives the depth at which the waves will break as a function of the volume. If the waves travel up a channel with a sloping bed, the larger waves break first, and the depths at which they break is proportional to the square root of their volunae.

The authors express their appreciation to Herbert N. Eaton of the National Bureau of Standards for his valuable criticisms and review of the paper.

\section{REFERENCES}

[1] Ph. Forchheimer, Hydraulik, [ed. 3] p. 295 (B. G. Teubner, Leipzig and Berlin, 1930).

[2] H. Favre, Etude Théorique et Expérimentale des Ondes de Translation dans les Canaux Découverts (Dunod, Paris, 1935).

[3] P. Massé, Hydrodynamique Fluviale Régimes Variables (Hermann, Paris, 1935).

[4] H. Lamb, Hydrodynamics, [ed. 5] (Cambridge University Press, Cambridge, England, 1924). 
[5] J. Boussinesq, Thérie des ondes et des remous qui se propagent le long d'un canal rectangulaire horizontal, en communiquant au liquide contenu dans ce canal de vitesses sensiblement pareilles de la surface au fond, Liouville's J. Math. 1\%, 55 (1872).

[6] H. Bazin, Recherche expérimentales sur la propagation des ondes, Mém. divers Savants a L'Acad. des Sci. 19, 495 (1865).

[7] J. Scott Russell, Report on Waves. Report of the fourteenth meeting of the Brit. Assn. Adv. Sci., p. 311. (John Murray, London, 1845.)

[8] J. Scott Russell, Report of the Committee on Waves. Report of the seventh meeting of the Brit. Assn. Adv. Sci., p. 417. (John Murray, London, 1838.)

[9] H. Bouasse, Houle, Rides, Seiches et Marées (Delagrave, Paris, 1924).

[10] J. Boussinesq, Essai sur la théorie des eaux courantes, Mém. divers Savants a L'Acad. des Sci. 32, 56 (1891).

[11] D. J. Korteweg and G. de Vries, On the change of form of long waves. Phil. Mag. [5] 39, 422 (1895).

[12] G. G. Stokes, Mathematical and Physical Papers, 1 (Cambridge University Press, Cambridge, England, 1880).

[13] J. McCowan, On the solitary wave, Phil. Mag. [5] 32, 56 (1891).

WASHINGTON, October 24, 1939. 